\title{
Variazione della verticale apparente e sismicità nella zona di Tolmezzo $\left(^{*}\right)$
}

\author{
P. Calor - G. Romualdi
}

Ricevuto il 10 Novembre 1964

\begin{abstract}
RIASSUNTO. - Si è già accennato, in precedenti pubblicazioni, all'esistenza di una stretta correlazione fra alcuni fenomeni sismici nella zona di Tolmezzo e le variazioni della verticale apparente, in detta zona registrate. Qui si riportano, in dettaglio, ulteriori testimonianze a questo riguardo, soffermandosi altresi sull'c inquietudine microsismica ", sovente associata ai periodi di forte variazione della verticale del luogo.

La recente entrata in servizio di una nuova, ottima stazione sismica a Somplago (Lago di Cavazzo), presso la centrale in roccia dell'ENEL, ha consentito l'intensificazione del controllo sismologico della zona, che servirà a meglio precisarne le caratteristiche dinamiche. Fra l'altro, è stato possibile valutare l'effetto delle stratificazioni sopportanti le stazioni d'osservazione sull'ampiezza delle registrazioni sismiche (l'« Untergrundtaktor" di Beno Gutenberg): a Tolmezzo, infatti, le vibrazioni relative ai terremoti vicini, o alla fase iniziale dei terremoti di lontana origine, subiscono -- nei confronti di Somplago - una notevole amplificazione locale, dovuta alla sottostante coltre alluvionale del Tagliamento.
\end{abstract}

SUMMARY. - In former papers, the authors have already mentioned the presence of a close connection between certain seismic phenomena in the area of Tolmezzo and the variations of the apparent vertical recorded in such area. The present papers reports in detail further information on this point, emphasizing also the "microseismic restlessness", often connected with periods of strong variation of the local vertical.

The recent beginning of the activity of a new and very well-equipped seismic station at Somplago (Lake of Cavazzo), at the central in rock built of the ENEL, has made it possible to intensify the seismological control of the area, which will be useful for a better definition of its dynamic charac. teristics. It has also been possible to evaluate the effect of the stratifica-

(*) Questo lavoro è stato condotto con contributi del Consiglio Nazionale delle Ricerche. 
tions which bear the observation stations on the amplitude of the seismic recordings (the " Undergroundfactor" mentioned by Beno Gutenberg): at Tolmezzo, in fact, the vibrations related to nearby earthquakes or to the initial phase of far earthquakes undergo - as compared to somplago a remarkable local amplification, due to the underlying alluvial stratum of the Tagliamento river.

1. - È grià stato accennato, in precedenti lavori, all'evidente correlazione fra lenti movimenti della crosta terrestre e alcuni terremoti locali nella zona di Tolmezzo. I risultati più cospicui delle indagini già compiute sono stati riassunti in pubblicazioni del $1958\left(^{1}\right)$ e del $1962\left(^{2}\right)$.

Esse furono condotte usufruendo di stazioni clinografiche (Fig. 1) funzionanti sulle rive dell'Ambiesta (affluente di destra del Tagliamento, presso Tolmezzo) e della stazione sismica di Tolmezzo.

Dal 1957 questa stazione mancava della componente verticale. Nel caso di scosse locali di piccola intensita (che furono la quasi totalità degli ultimi anni), non registrate quindi da altre stazioni sismiche, riusciva impossibile - anche in mancanza di notizie macrosismiche - precisare il loro epicentro. Dal Gennaio 1963 è entrata in funzione un'ottima stazione sismica a Somplago (sul lago di Cavazzo), fornita di apparecchi elettromagnetici Girlanda, a breve periodo. Ciò ha permesso di localizzare le zone epicentrali di molte scosse locali avvenute in tale anno. Inoltre, dal Novembre 1963, la componente E-W Girlanda di Tomezzo, è stata affiancata da una componente verticale dello stesso tipo.

La nuova dotazione strumentale, permette ora di individuare il luogo di provenienza delle piccole scosse locali. Ciò riveste notevole interesse, ai fini della microsismicità della zona intorno a Tolmezzo e delle sue principali caratteristiche. Solo d'ora innanzi, infatti, sarà possibile stabilire quali settori, sismicamente attivi, influenzano altresi la verticale apparente e quali entrano in stato di inquietudine, atta a determinare quella particolare agitazione microsismica, rilevata talvolta a Tolmezzo, e alla quale è stato dato il nome di "inquietudine microsismica" ( $\left.{ }^{2}\right)$.

Nelle pagine che seguono, sono riassunti i risultati delle osservazioni relative agli anni 1960-1963.

2. - L'osservazione delle registrazioni clinografiche ottenute presso la diga dell'Ambiesta negli anni 1959-1963 mostra innanzi tutto una netta differenza di comportamento, peraltro già messa altre volte in evidenza, fra i clinografi situati in sponda destra e quelli in sponda sinistra, a testinonianza dell'esistenza di movimenti contrastanti lungo una linea di 


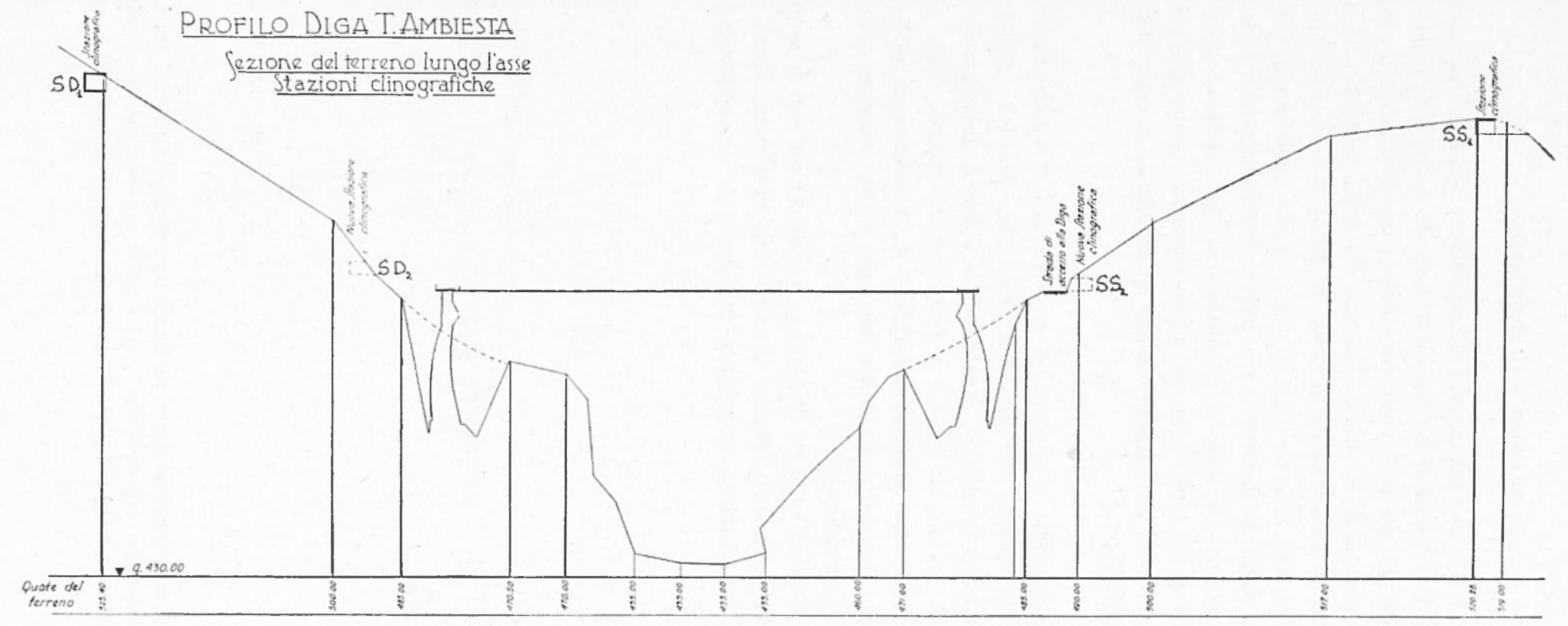

Fig. 1. - Posizione delle stazioni clinografiche presso la diga dell'Ambiesta (nella sezione della valle, figurano tratti della diga di sbarramento). 
frattura situata fra le due postazioni clinografiche. Nel periodo esaminato, mentre in sponda sinistra si ha un alternarsi di periodi di quiete clinografica, caratterizzati da deviazioni pressoché insignificanti della verticale apparente, con periodi di sensibili spostamenti della verticale, in sponda destra si registrano solo lentissimi e piccolissimi movimenti, propri dei periodi di quiete, con ritorno della verticale nella posizione di partenza.

Per quanto riguarda il movimento della verticale apparente in sponda sinistra si può notare che esso si manifesta in due modi: o come movimento, sensibile si, ma lento e in direzione costante anche per più mesi, oppure come serie di movimenti ampi e convulsi, spesso solo sulla componente NW-SE.

3. - Analogamente a quanto si osserva nelle registrazioni clinografiche presso la sponda sinistra dell'Ambiesta, anche le registrazioni sismiche di Tolmezzo mostrano, per l'attività sismica di carattere locale, un'alternanza di periodi di quiete e di maggior attività. Tale attività inoltre può assumere due aspetti; essa infatti si manifesta sia sotto forma di scosse di varia intensità, sia come agitazione minutissima irregolare, denominata "inquietudine ».

L'attività sismica sembra dunque in correlazione con i movimenti della verticale apparente in sponda sinistra dell'Ambiesta, come risulta meglio da una osservazione congiunta dei due tipi di registrazione.

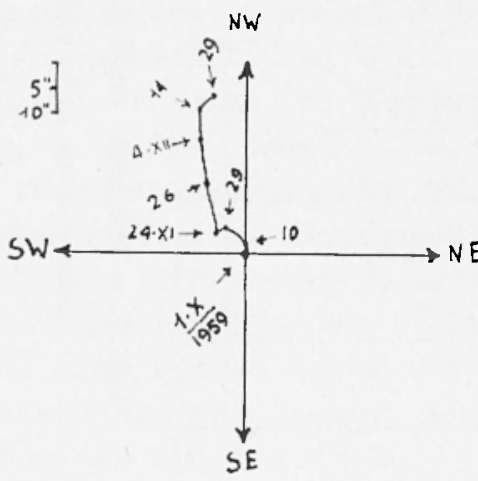

$5 S_{1}$

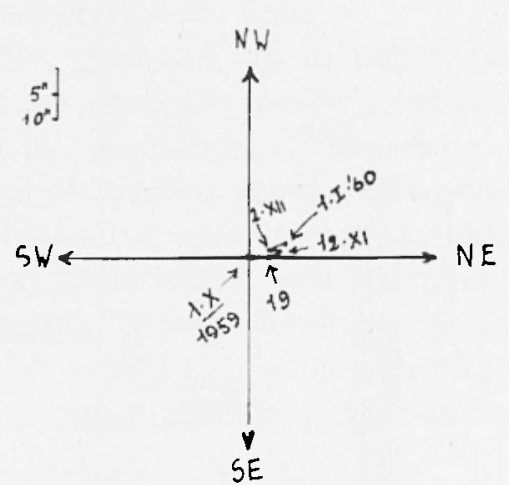

$5 D_{1}$

Fig. 2. - Movimenti angolari della verticale apparente registrati sulla sponda sinistra (postazione clinografica $S S_{1}$ ) e sulla sponda destra (postazione clinografica $\$ D_{1}$ ) nel periodo dal 1.X.1959 al 1.I.1960. 

4. - Alla fine di Novembre del 1959 la postazione clinografica sulla sponda sinistra dell'Ambiesta registra una netta deviazione della verticale apparente, circa in direzione $\mathrm{NW}$ (Fig. 2); tale movimento prosegue, seppure meno accentuato, per tutto Dicembre e sembra sfociare in una serie di scosse locali, registrate a Tolmezzo nei giorni 6, 7 e 9 Gennaio 1960. La serie di scosse sembra porre termine ad una crisi tettonica della regione, in quanto dopo il 9 Gennaio i clinografi si acquietano.

In realtà la quiete è solo momentanea, giacché fin dai primi di Febbraio lo spostamento della verticale apparente riprende in maniera notevole, però in direzione all'incirca $\mathrm{E}$ (Figg. 3 e 4). Si deve perciò ritenere che il contrasto delle tensioni in atto nella zona, non si sia esaurito ma piuttosto trasferito in altra direzione. Il perdurare di uno stato di tensione è anche indicato dal ripetersi di scosse, seppure molto deboli, nel mese di Gennaio; va peraltro messo in evidenza che l'attività sismica diminuisce gradualmente fino a cessare nei mesi di Aprile, Maggio e Giugno. Contemporaneamente a questo periodo di quiete sismica, la verticale apparente accusa una deviazione costante dapprima in direzione $\mathrm{E}$, quindi NE e da metà Maggio all'incirca in direzione ESE. Tale deviazione che assume direzione $\mathrm{E}$ si accentua nel mese di Giugno e prosegue fino a metà Luglio, quando la direzione muta bruscamente di circa $90^{\circ}$; quasi contemporaneamente si registra a Tolmezzo $\left(14 / \mathrm{VII} / 60,05^{\mathrm{n}} 15^{\prime}\right.$ ca) una forte scossa, a cui seguono delle ripetizioni nello stesso giorno e nei giorni successivi (Figg. 4, 5, 6 e 7).

Il fenomeno sembra identico a quello verificatosi fra il Dicembre $\mathbf{1 9 5 9}$ e Gennaio 1960; anche in questo caso il contrasto delle tensioni non si esaurisce con la serie di scosse ma si trasferisce in un'altra direzione. Ciò è dimostrato, oltre che dal perdurare dello spostamento della verticale apparente, anche dal ripetersi di scosse per tutto il mese di Agosto.

Nei mesi successivi le variazioni della verticale apparente diventano addirittura convulse tanto da non poter essere chiaramente interpretate fino al mese di Febbraio 1961 (Fig. 8).

A prima vista, il movimento della verticale sembrerebbe risolversi in una serie di bruschi e rapidi movimenti in direzione $\mathrm{NW}$ con successivo ritorno alla posizione di partenza, intervallati da brevi periodi di calma assoluta. In realtà, da quanto è possibile vedere fra Febbraio e Marzo, il movimento della verticale deve essere considerato come la risultante di due tipi di movimenti sovrapposti (Fig. 9): un movimento costante di base, che determina una deviazione permanente in direzione WNW (e che quindi prosegue il movimento verificatosi in altra direzione nel '60), 


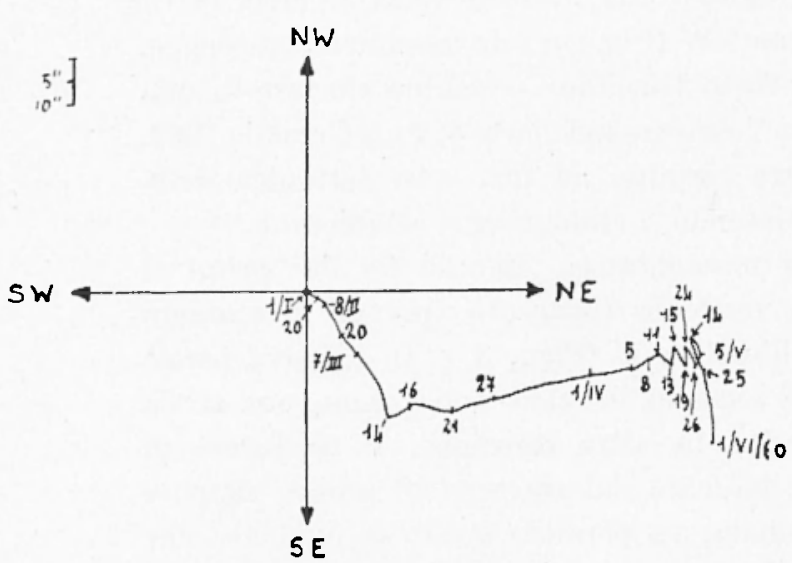

Sponda sinistra

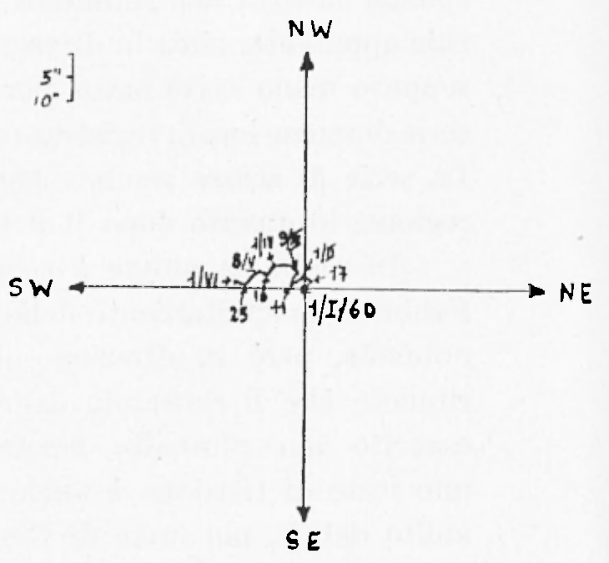

Sponda destra

Fig. 4. - Risultante dei movimenti angolari della verticale apparente in sponda sinistra e in sponda destra nel periodo dal l.I.1960 al l.VI.1960. Si noti la soverchiante attività della sponda sinistra nei confronti della sponda destra.

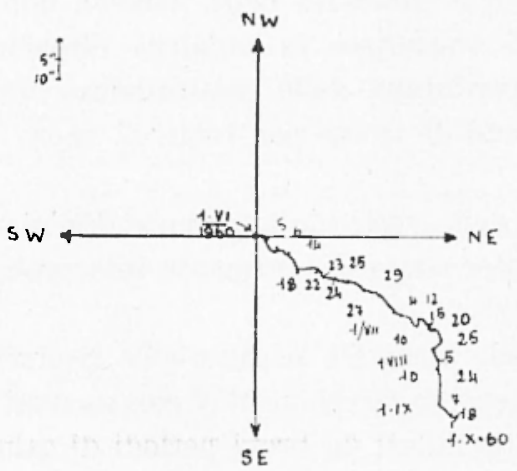

Sponda sinistra

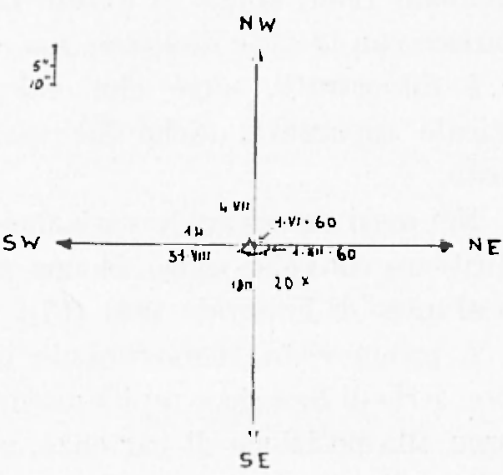

Sponda destra

Fig. 5. - Risultante dei movimenti angolari della verticale apparente in sponda sinistra e in sponda destra nel periodo fra il 1.VI.1960 e il 1.XII.1960; da notare la netta differenza di entità di movimento nelle due postazioni. 


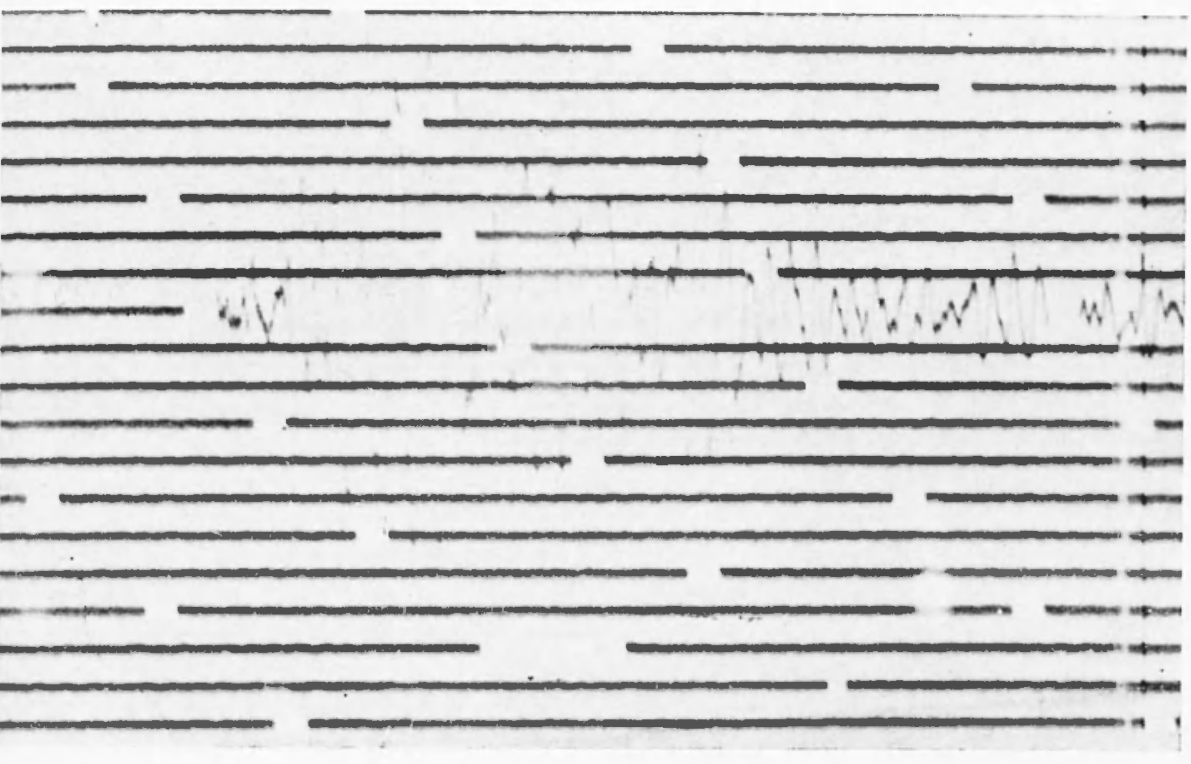

Fig. 6. - Stazione sismica del Vajont: comp. vert. - registrazione della scossa di Tolmezzo del 14. VII. $1960\left(05^{\mathrm{h}} 18^{\mathrm{m}} ; \Delta t=-7^{\mathrm{s}}, 6\right)$.

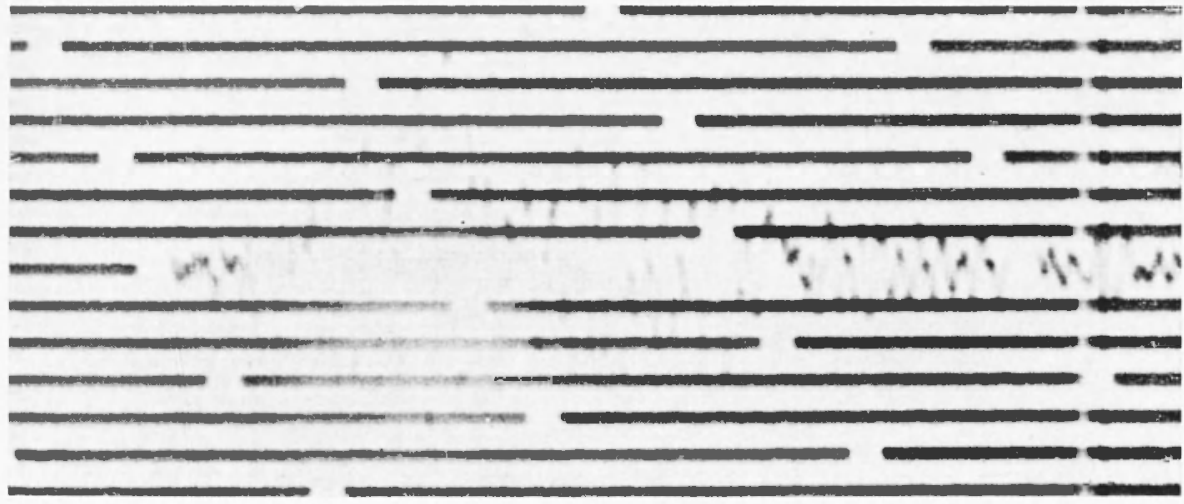

Fig. 6a. - Comp. N-S. 


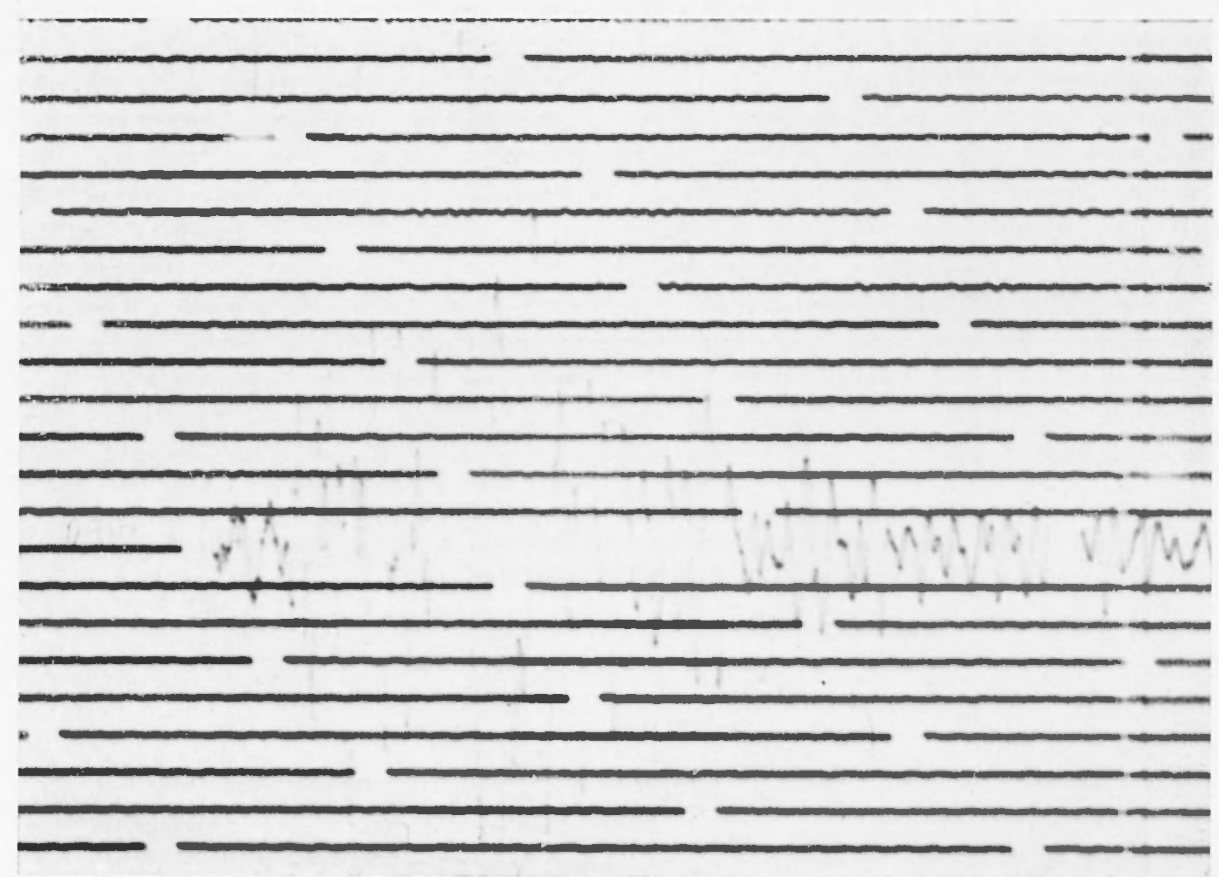

Fig. 6b. - Comp. E-W. 


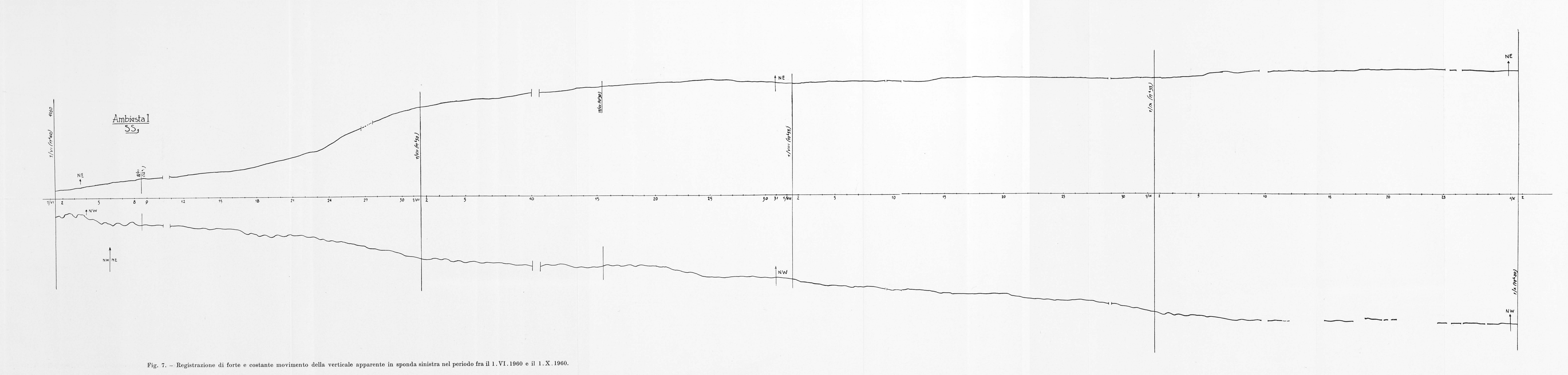




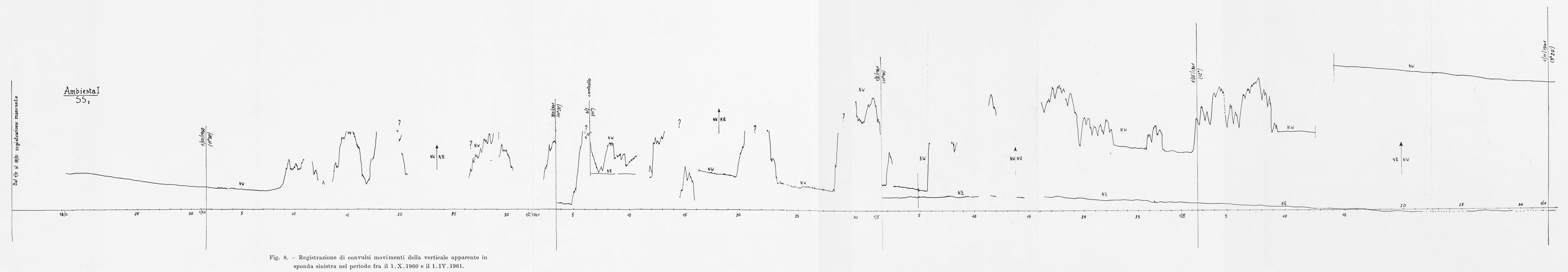



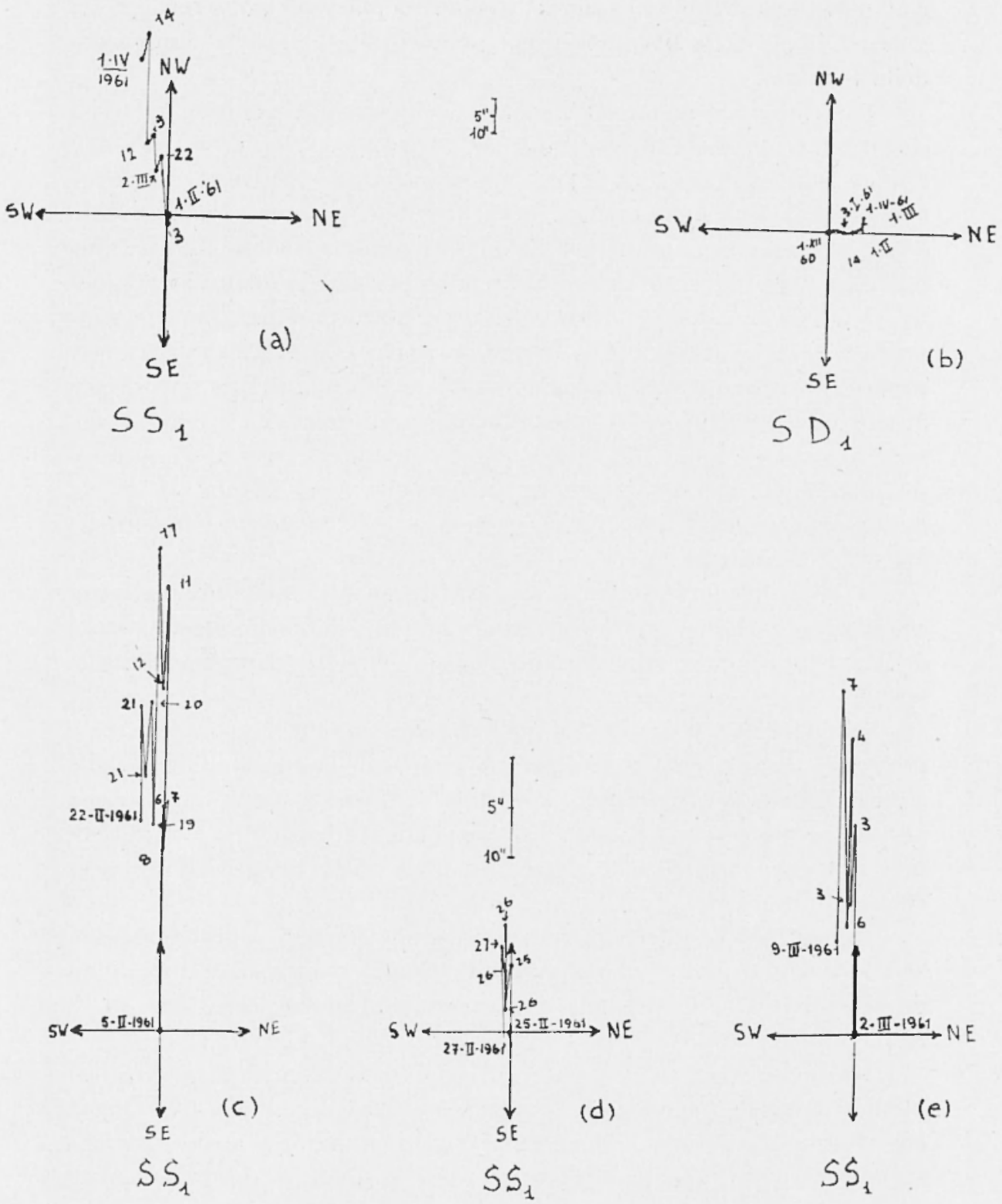

Fig. 9. - Risultante dei movimenti angolari della verticale apparente in sponda sinistra e in sponda destra nel periodo fra il 1.X.1960 e il l.IV.1961; in sponda sinistra a un movimento costante di base (a), si sovrappone un movimento rapido e improvviso, pressoché esclusivamente lungo l'asse $\mathrm{NW}(c),(d)$ ed $(e)$. 
e un movimento rapido e saltuario, ripetentesi più volte anche nella stessa giornata lungo l'asse NW. ma che non produce una deviazione permanente della verticale.

Contemporaneamente all'agitazione clinografica, perdura l'attività sismica con numerose scosse (Figg. 10, 11 e 12), e si ha l'insorgere dell'inquietudine sismica (Fig. 13) che appare nel mese di Ottobre, raggiunge il suo aspetto più vistoso nel mese di Dicembre, va quindi gradualmente scemando nei mesi di Gennaio e Febbraio e termina a metà Marzo. Dopo tale data si può dire che la zona entri in un periodo di quiete che perdura fino a Ottobre. Infatti, in tutto questo periodo la verticale ha solo normali lentissime variazioni (Fig. 14) mentre anche l'attività sismica è molto ridotta: si hanno saltuarie scosse non molto forti, o addirittura sotto forma di tracce, e rare tracce d'inquietudine nei primi mesi. Fa eccezione una serie di quattro scosse, di cui una molto forte fra il 19 e il 27 Agosto. Ad ogni modo, in tutto il periodo da Agosto a metà Ottobre, non solo manca ogni traccia d'inquietudine ma non si riscontra neanche la normale attività microsismica.

A metà Ottobre riprende improvvisamente l'inquietudine sismica che si ripete anche nei mesi successivi, seppure in modo meno vistoso dell'anno precedente. Minori sono anche il numero e l'intensità delle scosse.

L'agitazione clinografica riprende bruscamente a metà Novembre e prosegue, anche questa in modo meno vistoso dell'anno precedente, fino a metà Marzo. In tutto questo periodo la verticale ha forti, improvvisi e saltuari movimenti in direzione $\mathrm{NW}$ che si impostano su un movimento di base pressoché insignificante e paragonabile a quello dei periodi di quiete (Fig. 15).

Mentre dunque ad un'agitazione clinografica meno accentuata rispetto al 60-61 corrisponde una inquietudine sismica ridotta, l'assenza di un movimento costante, sensibile della verticale coincide con l'assenza di forti scosse locali.

Da metà Marzo ai primi di Ottobre 1962, la zona attraversa un periodo di quiete: la verticale apparente ha solo normali lente variazioni con ritorno alle posizioni di partenza (Fig. 16), mentre le scosse sismiche si riducono a pochi deboli esempi fra la fine di Luglio e i primi di Agosto e a una scossa abbastanza forte localizzabile a Villa Santina.

In tutto il periodo l'attività microsismica è normale o molto ridotta.

Ai primi di Ottobre 1962, l'attivistà sismica riprende, peraltro con anticipo rispetto a quella clinografica, con una leggera inquietudine, per- 


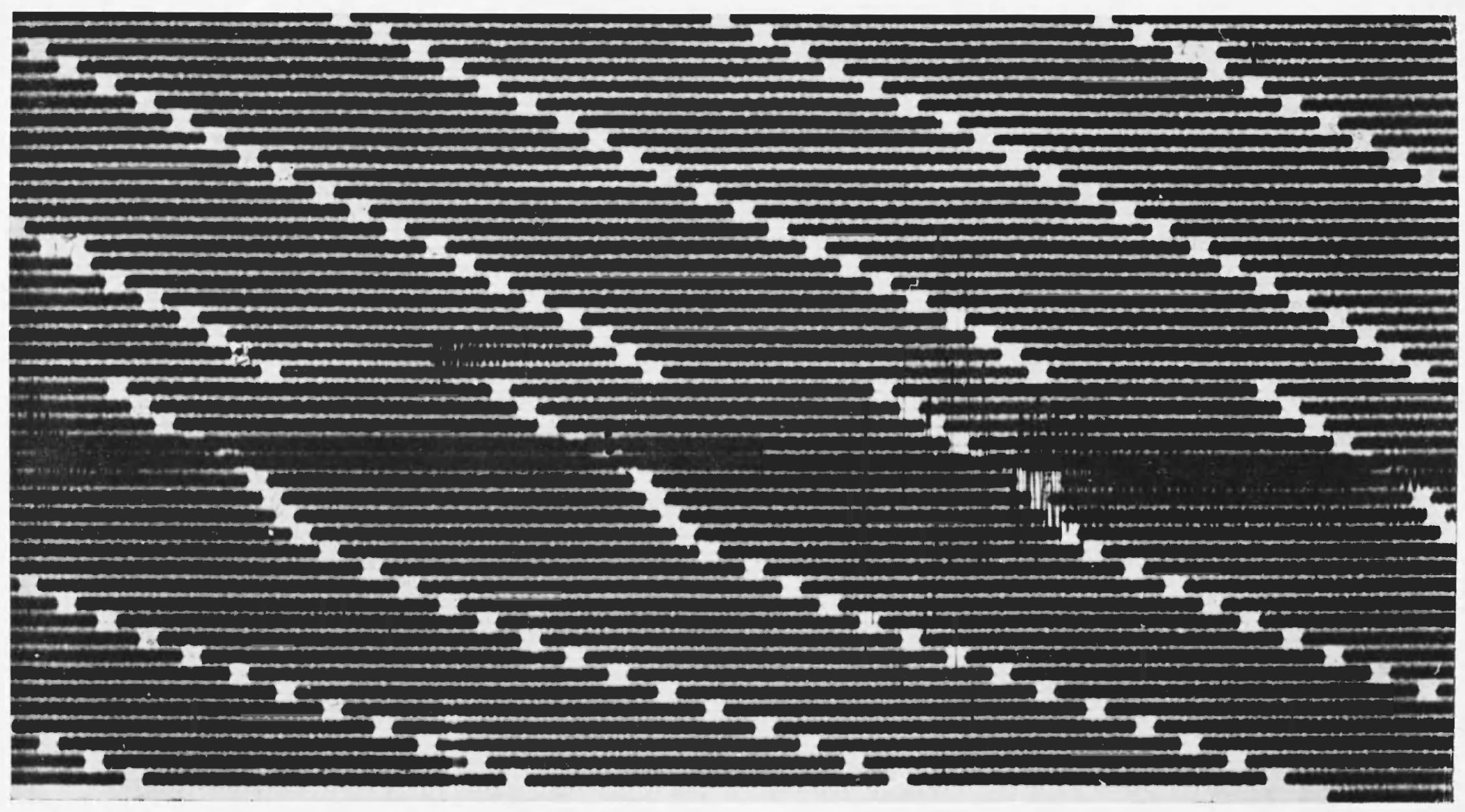

Fig. 10. - Stazione sismica di Tolmezzo, comp. EW; registrazione di scosse locali (26.X.1960), in periodo di forte agritazione clinografica presso l'Ambiesta. 


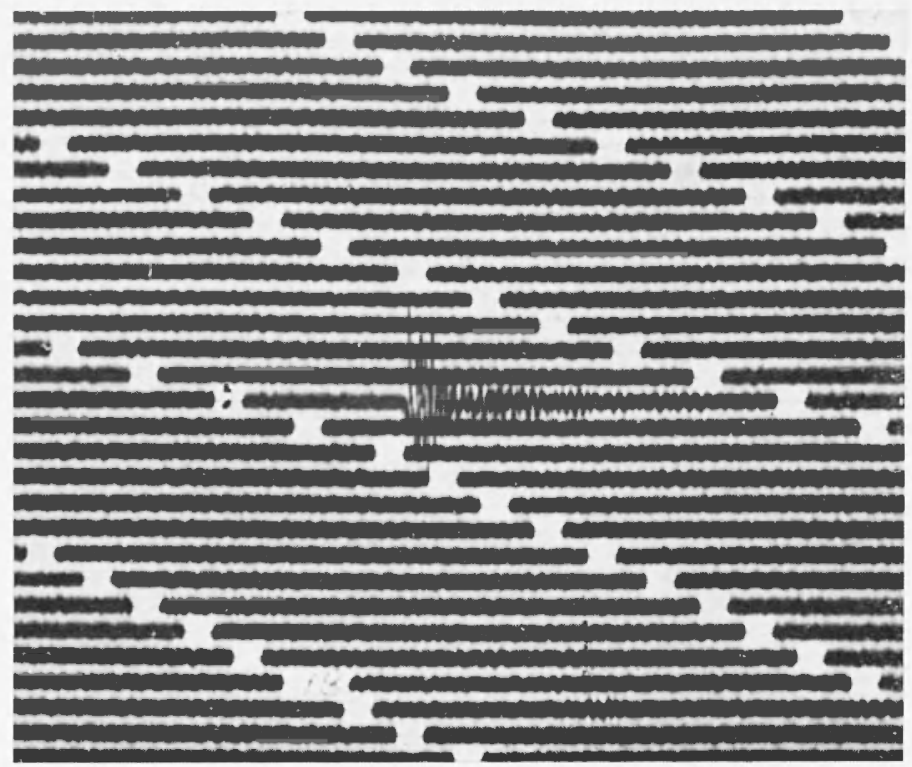

Fig. 11. - Stazione sismica di Tolmezzo, comp. E-W; registrazione di una scossa locale del $26 . X .1960\left(20^{\mathrm{n}} 57^{\mathrm{m}}\right.$ ca. $)$.

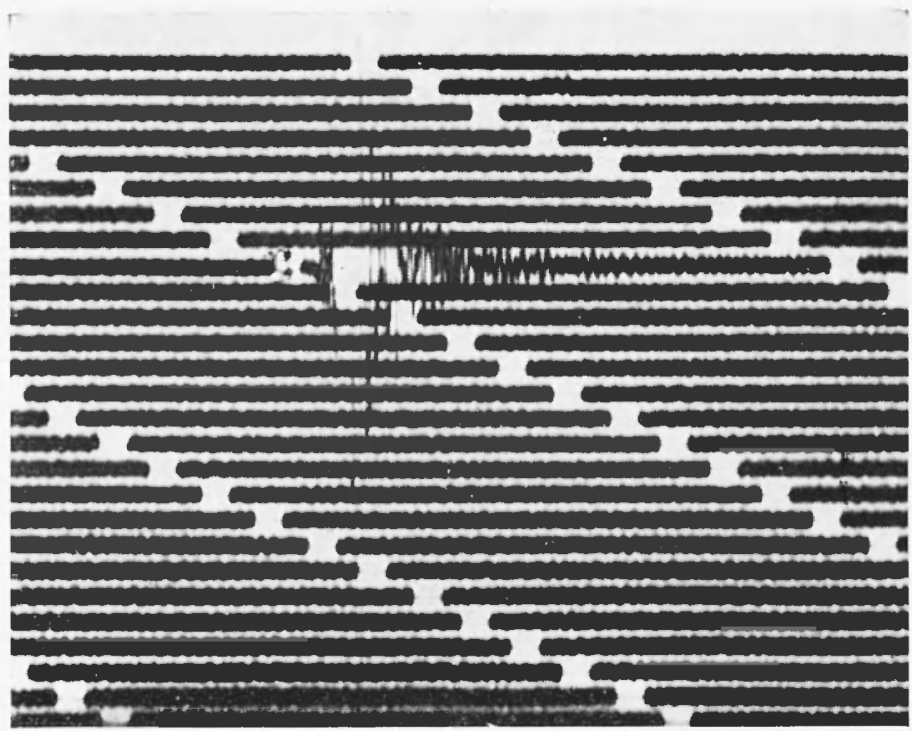

Fig. 12. - Stazione sismica di Tolmezzo, comp. E.Ws registrazione di una scossa locale del $27 . X .1960\left(05^{\mathrm{h}} 30^{\mathrm{m}}\right)$, sempre in periodo di notevoli variazioni della verticale apparente. 


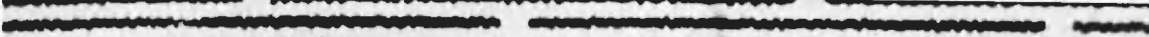
-

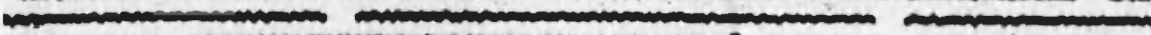

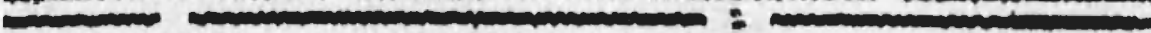

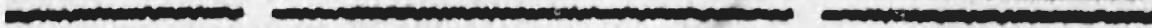

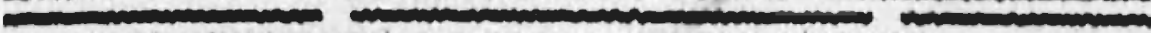

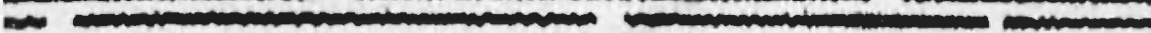

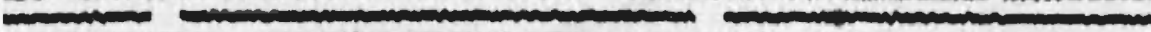

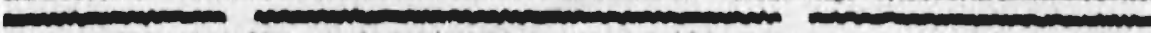

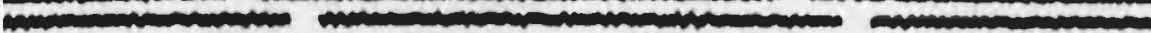

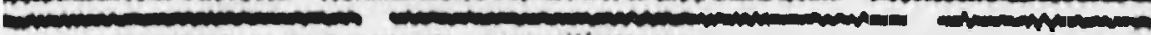

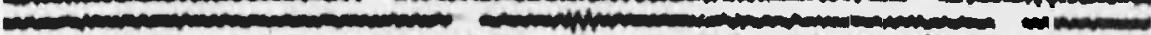

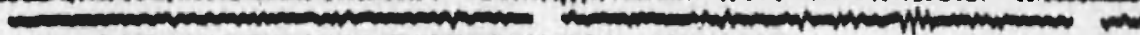

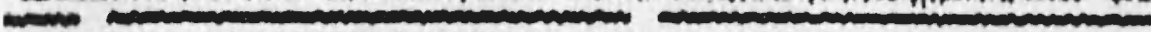

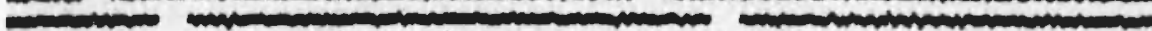

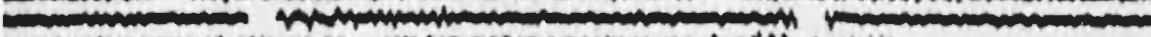

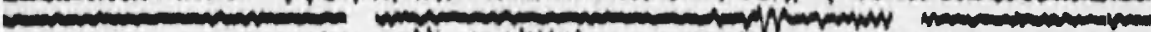

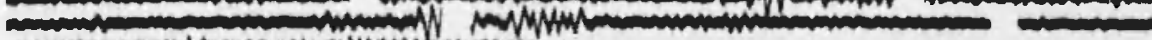

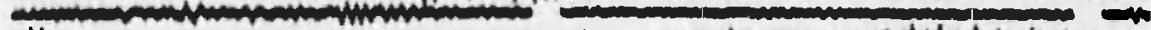

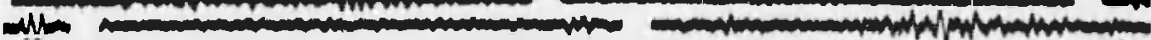
mivin a

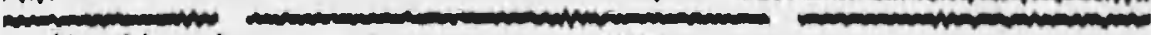

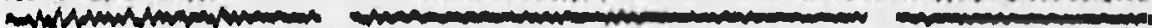

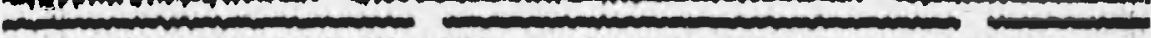

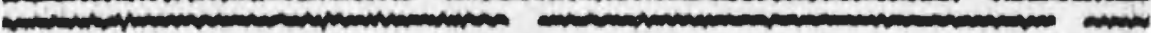

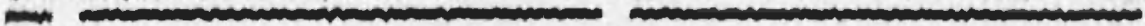
-

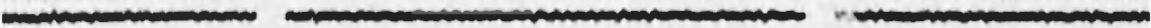
" "

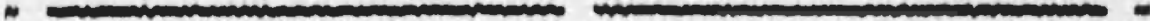

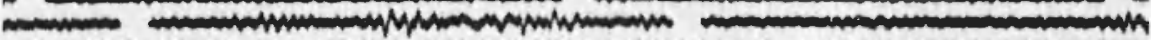
-

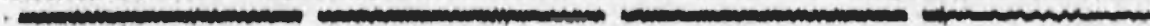

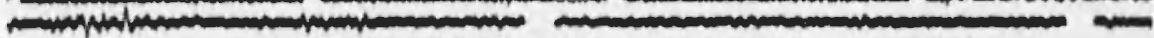
Dines E

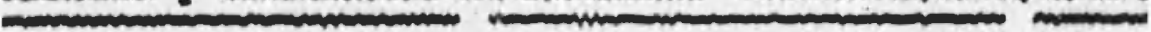
A A

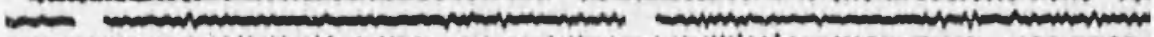

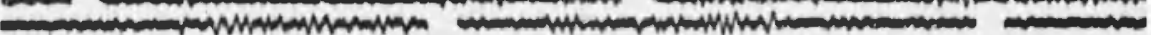

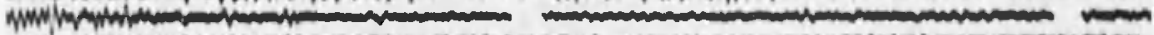
2017

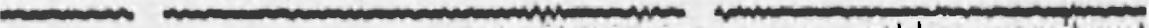

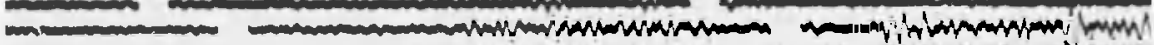

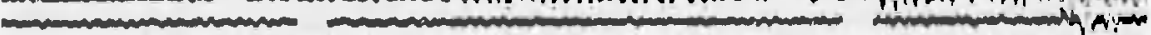

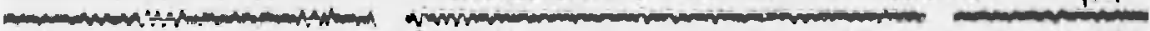

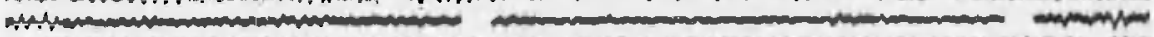

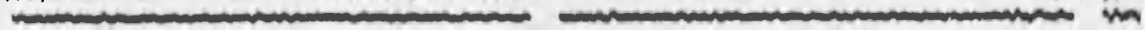

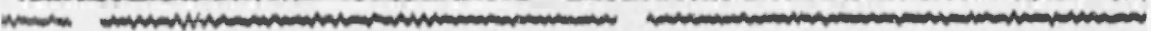

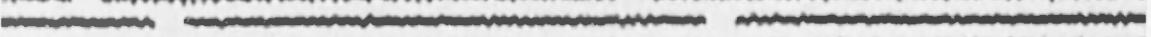

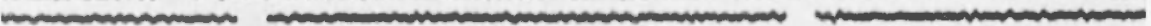

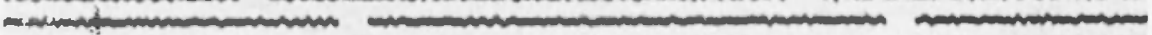
"

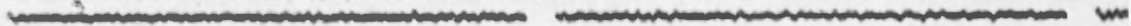

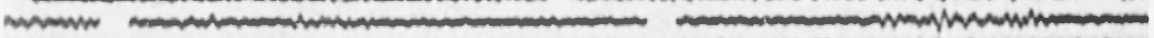

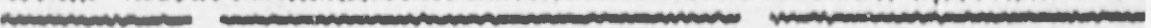

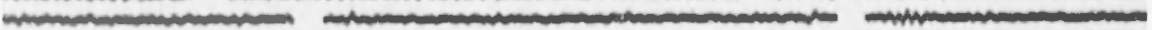
"

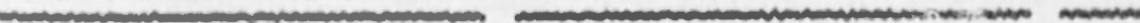

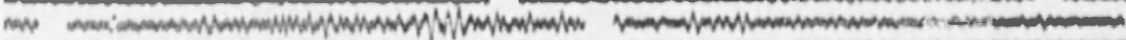

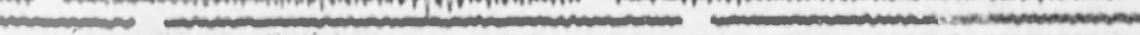

Fig. 13. - Stazione sismica di Tolmezzo, comp. E.W: esempio di registrazione d'inquietudine sismica nei giorni 6 e 7 Dicembre 1960 , associata a convulsa variazione della verticale apparente in sponda sinistra dell'Ambiesta. 
durante fino ai primi dieci giorni di Novembre; poi si ha normale attività microsismica. Ta in ogni modo detto che si hanno dubbi circa il perfetto funzionamento del sismografo in quel periodo.
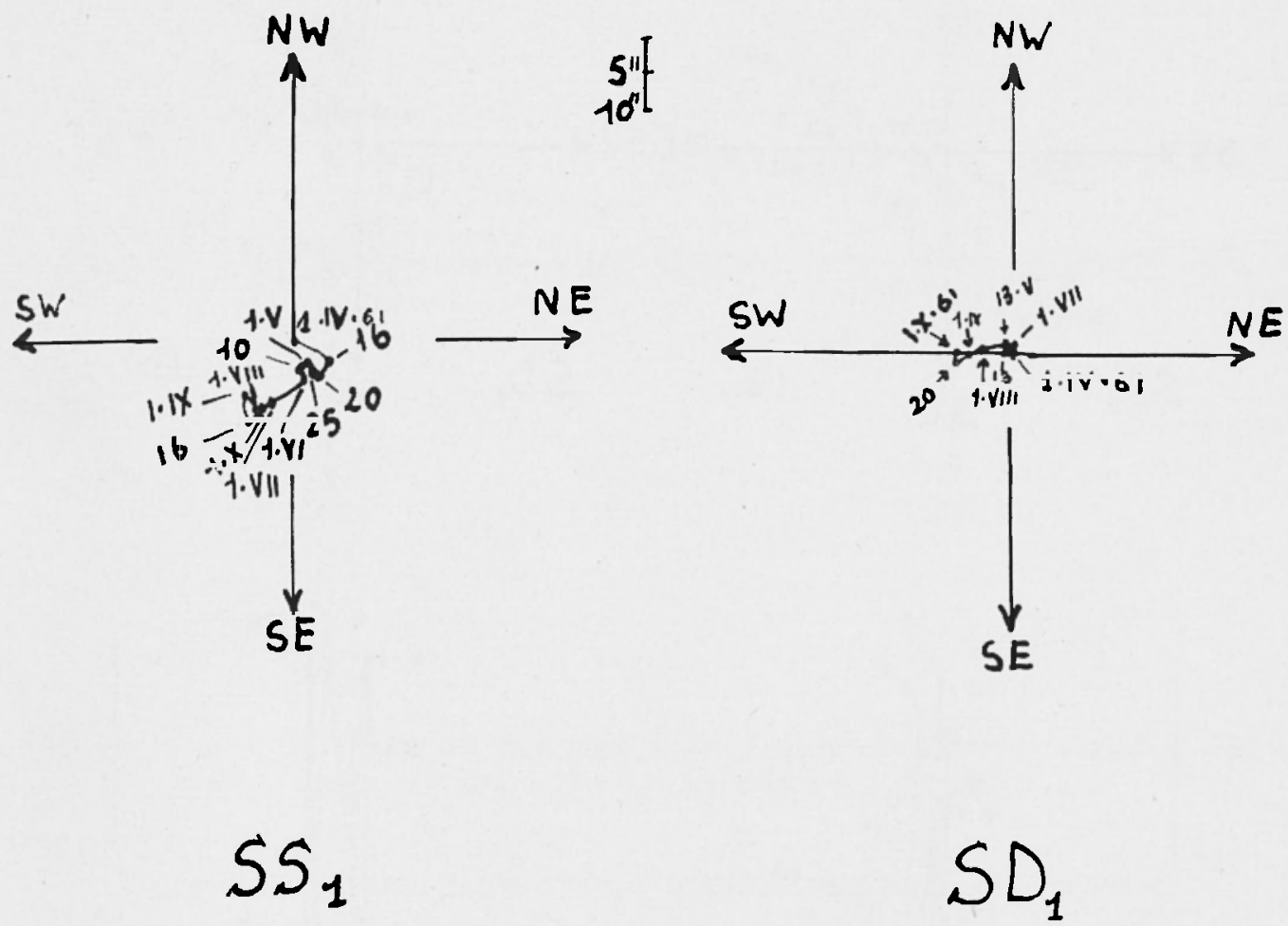

Fig. 14. - Risultante dei movimenti angolari in sponda sinistra e in sponda destra in periodo di quiete clinc rrafica (1.IV.1961 - $1 . X .1961$ ).

Nuovi segni di agitazione clinografica si cominciano ad avere verso $\mathbf{i}$ primi di Novembre con bruschi movimenti della componente NW, talvolta provocanti una variazione permanente sempre in direzione $\mathrm{NW}$. Tali movimenti si concentrano soprattutto nella seconda metà di Novembre e nella prima metà di Gennaio 63. Dopo quest'ultimo periodo la componente NW in spalla sinistra ritorna in quiete (Fig. 17).

Per quanto riguarda le registrazioni sismiche va notato che, dopo i segni d'inquietudine dell'Ottobre e Novembre, non si può notare una particolare traccia di attività da poter currelare all'inquietudine clinografica dell'inverno 62-63: si hanno infatti solo due scosse, peraltro deboli, in Dicembre e alcune scossette in Gennaio. Al contrario, un certo numero 

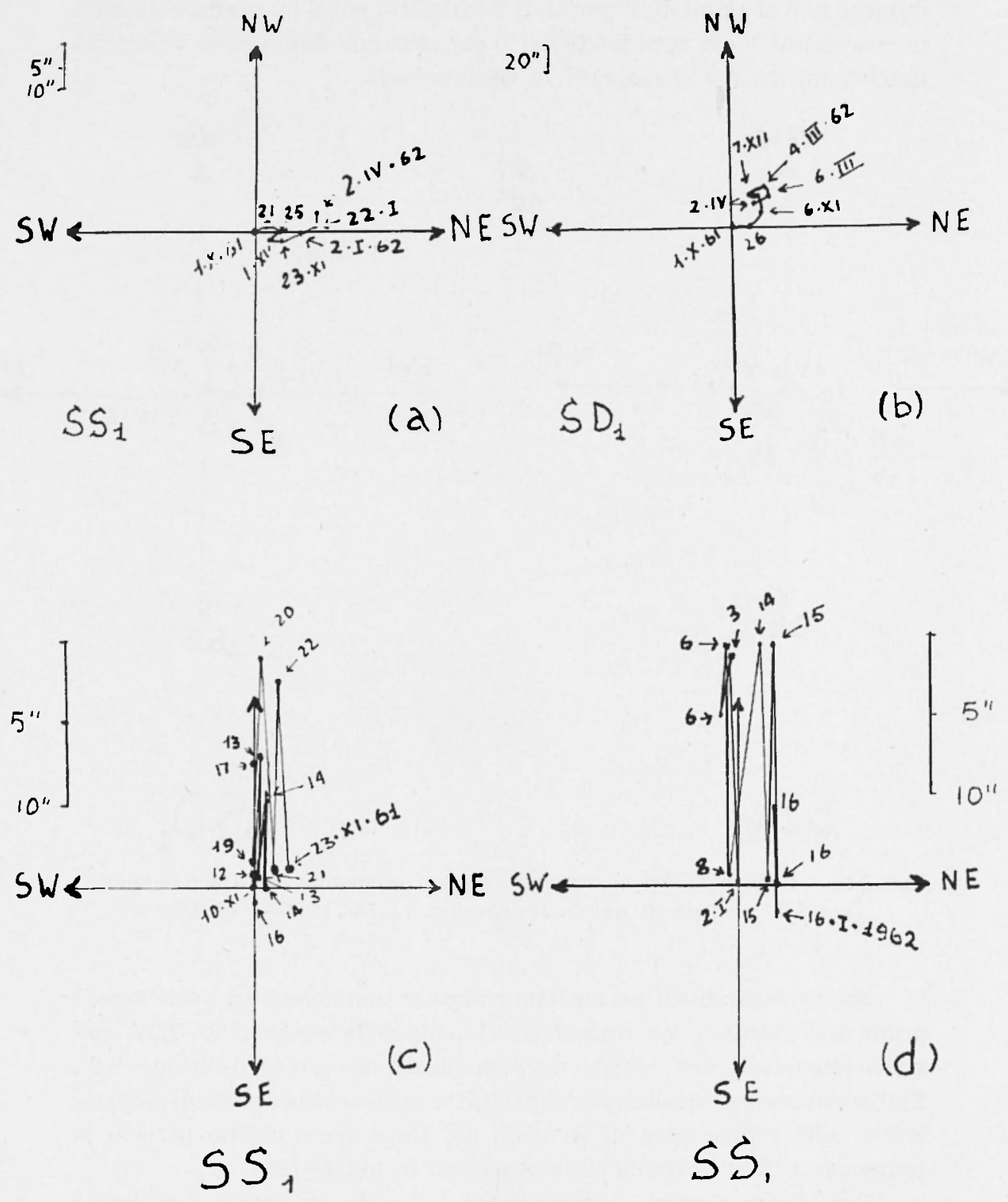

Fig. 15. - Risultante dei movimenti angolari della verticale apparente in sponda sinistra e in sponda destra fra il 1.X.1961 e il 2.IV.1962. In sponda sinistra, a un movimento di base molto ridotto (a), si sovrappongono bruschi e ripetuti movimenti lungo l'asse $\mathrm{NW}$ con ritorno della verticale alla posizione di partenza (c) e (d). 
di scosse, di cui alcune forti, si registrano nei mesi di Aprile, Maggio e Luglio, in cui i clinografi non registrano sensibili variazioni della verticale.
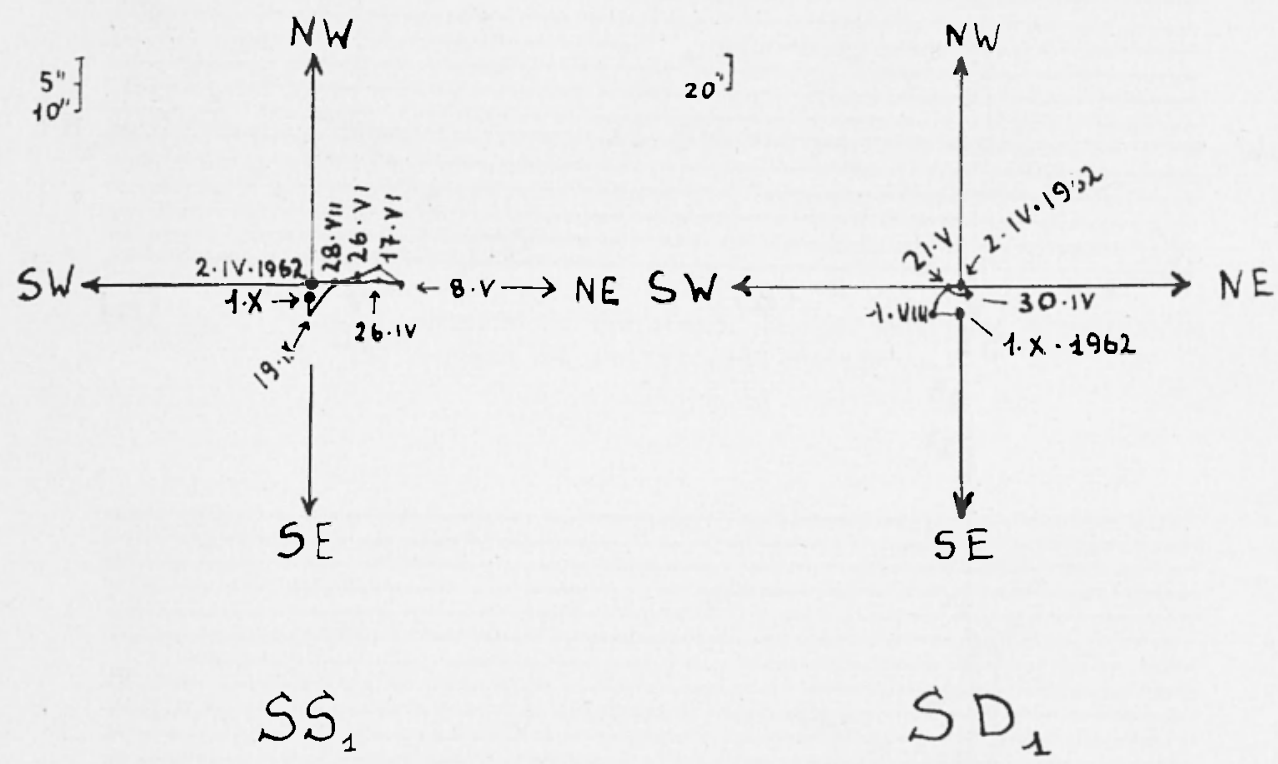

Fig. 16. - Risultante dei movimenti angolari in sponda sinistra e in sponda destra nel periodo fra il 2.IV.1962 e il 1.X.1962.

5. - Da quanto sopra visto, è possibile constatare quanto segue:

a) Le scosse sismiche sono generalmente precedute da periodi di lente ma sostanziali variazioni della verticale apparente nella regione epicentrale; e in ciò è possibile vedere il modo di reagire delle masse rocciose sotto l'azione di forze orientate, come sono quelle orogenetiche. Dapprima esse cedono lentamente, deformandosi plasticamente, poi sotto l'azione crescente delle tensioni pervengono al limite di rottura, con formazione di fratture e rilascio improvviso e istantaneo dell'energia accumulatasi nella prima fase.

b) L'inquietudine sismica, che riflette anch'essa una agitazione tettonica della regione, si accompagna in genere a rapide e brusche variazioni della verticale apparente. Essa può essere considerata come il manifestarsi di brevissimi, improvvisi movimenti degli strati rocciosi lungo linee di frattura. 


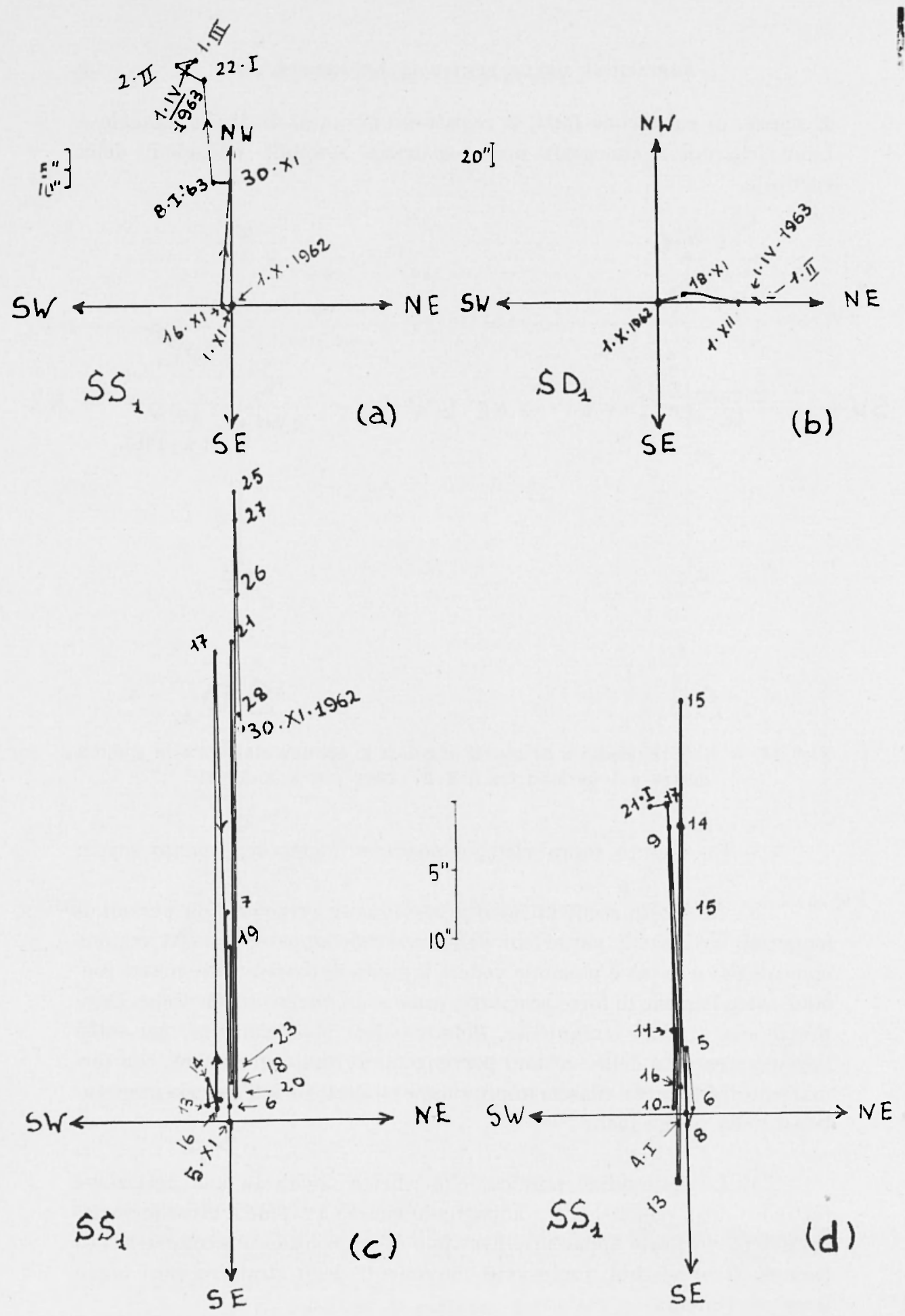

Fig. 17. - Risultante dei movimenti angolari in sponda sinistra (a) e in sponda destra (b), nel periodo fra il $1 . X .1962$ e il $1 . I V .1963$. In sponda sinistra la verticale apparente subisce improvvisi movimenti ripetentisi più volte lungo l'asse NW - (c) e (d) - che determinano un notevole spostamento complessivo (a). 


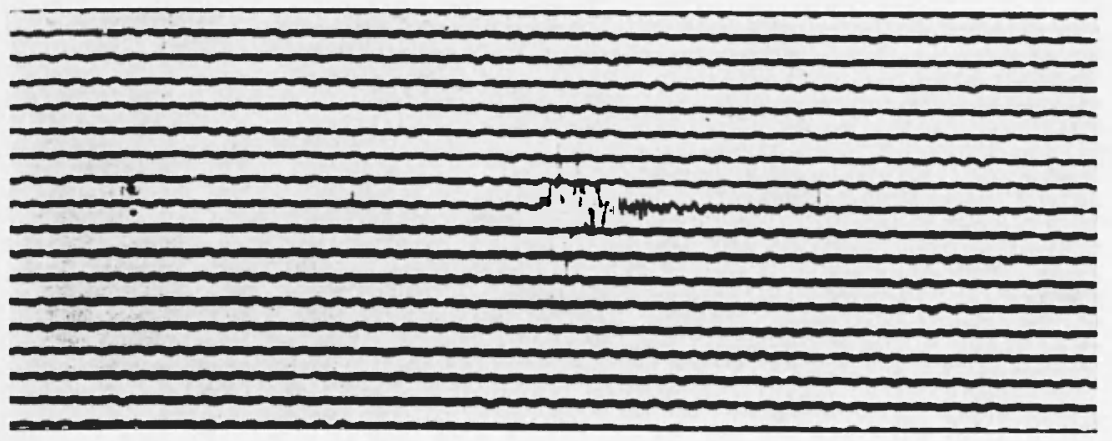

Fig. 18. - Stazione sismica di Somplago, comp. verticale: registrazione della scossa del 28 . VII. $1963\left(06^{\mathrm{h}} 12^{\mathrm{m}}\right.$ ('a.).

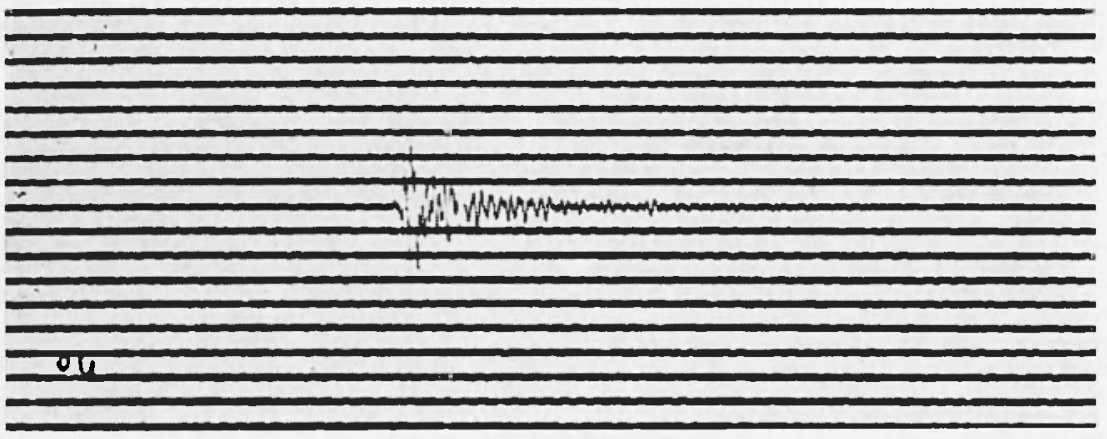

Fig. 18a. - Comp. SW-NE.

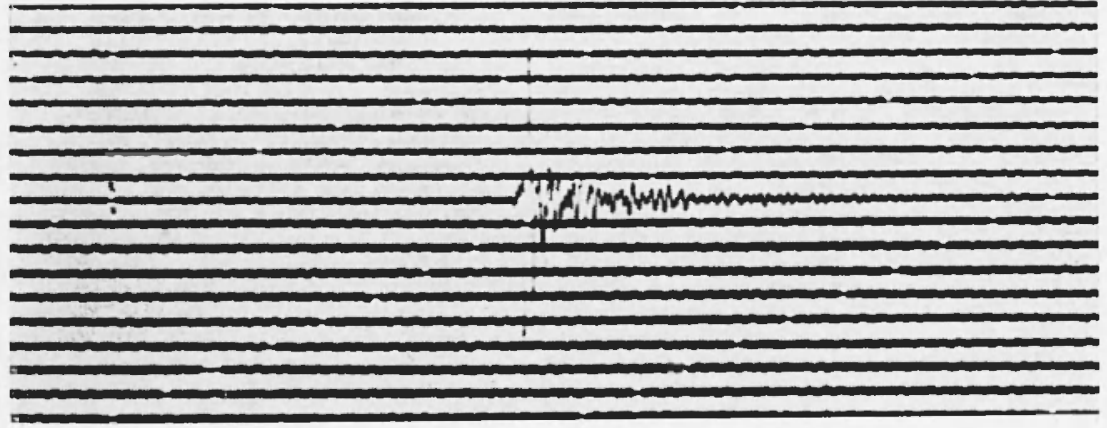

Fig. 18b. - Comp. SE-NW (epicentro come da Fig. 19). 


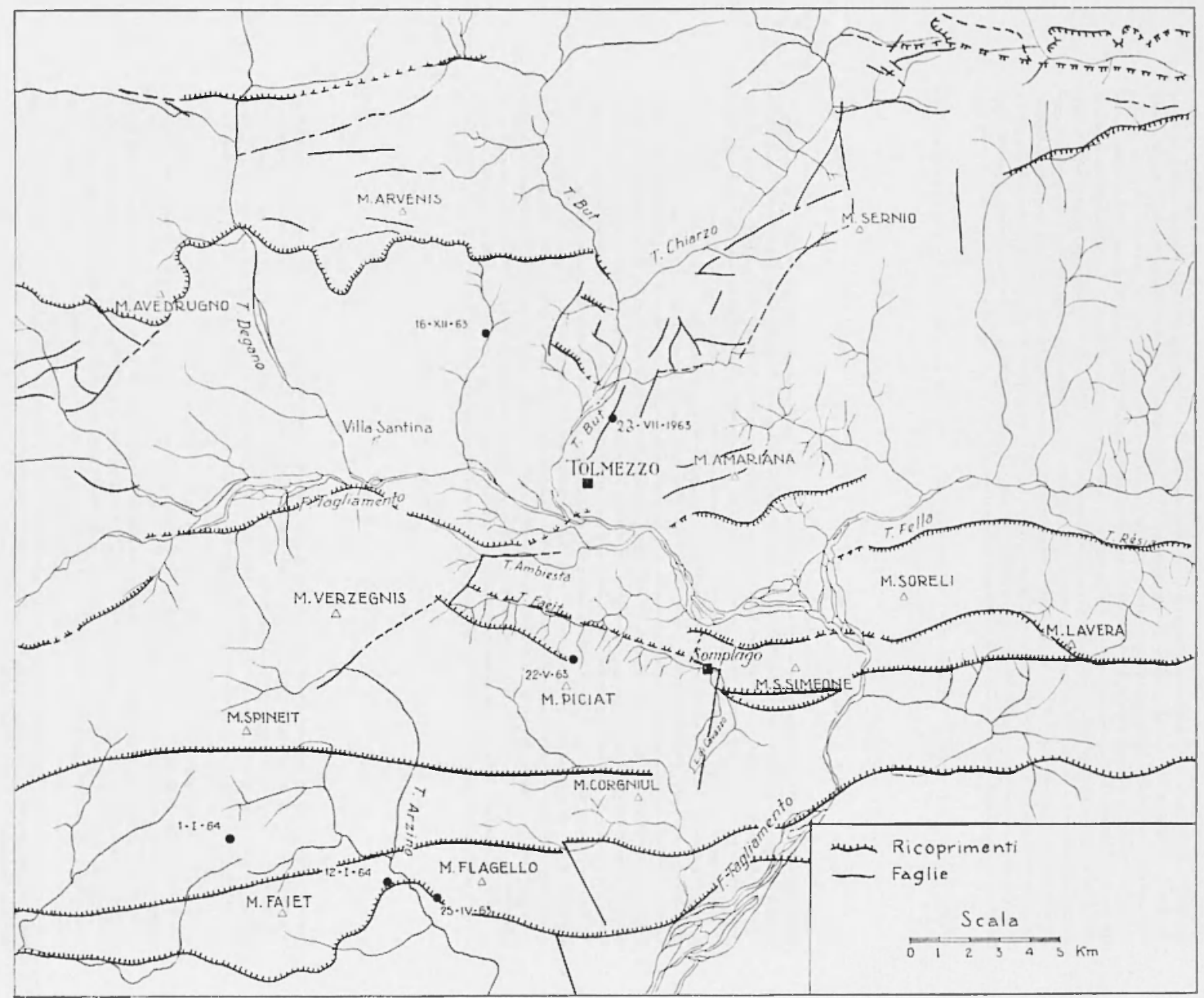

2
0
0
0
1
0
0
0
0
0
0

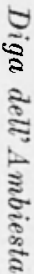

Fig. 19. - Principali linee di dislocazione tettonica o localizzazione di alcune recenti scosse. 
Fig. 20. - Scossa dell' 8 . I . 1963 $\left(17^{\mathrm{h}} 17^{\mathrm{m}}\right.$ ca.), registrata presso le stazioni di: Tolmezzo, comp. EW.

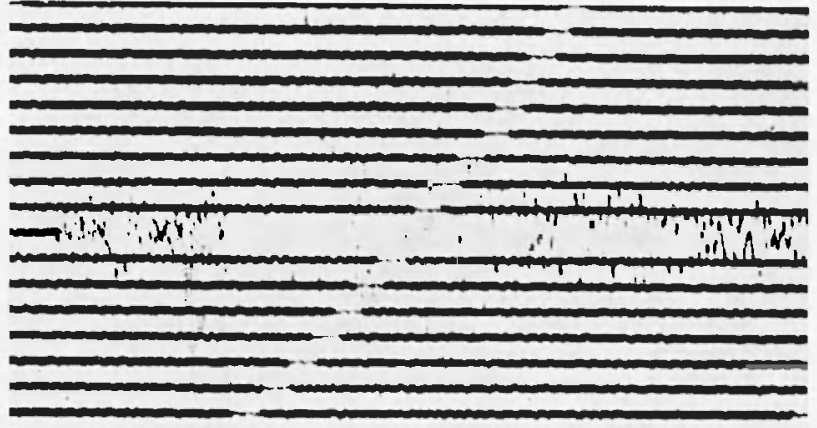

Fig. 20a. - Somplago, comp. vert. $(1=140$ km ca. da Som plago).

Fig. 20b. - Somplago, comp. SW-NE.
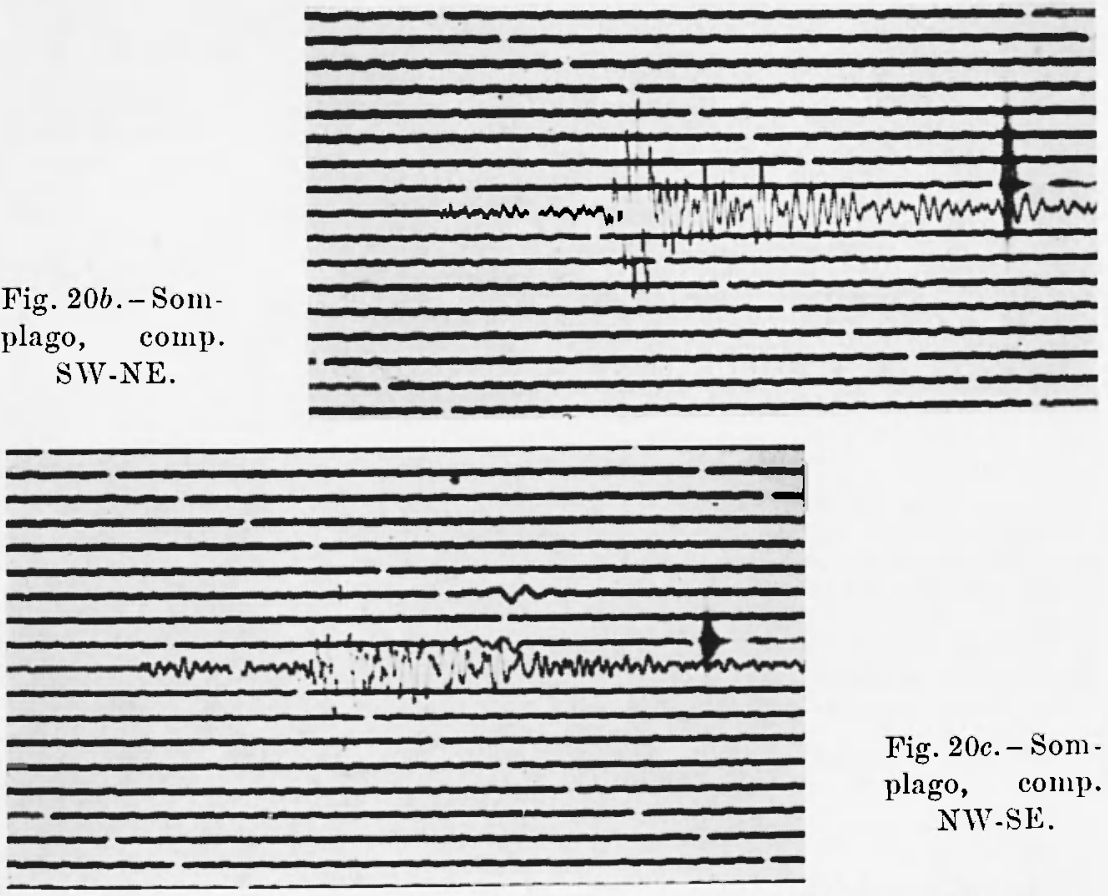

Fig. 20c. - Som plago, comp. NW.SE.

Si noti il forte fattore amplificante del sottosuolo di Tolmezzo (alluvione), nei confronti di quello di Somplago (roccia). 


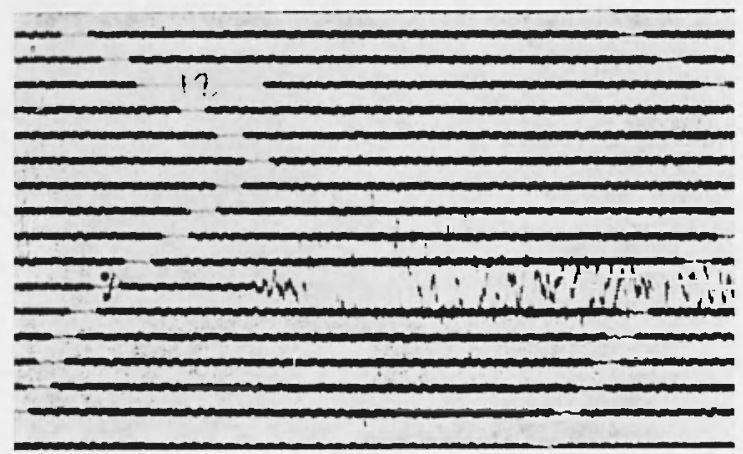

Fig. 21. - Scossa del 13.V. $1963 \quad\left(09^{\mathrm{h}} 52^{\mathrm{m}} \quad\right.$ ca.) registrata presso le stazioni sismiche di: Tolmezzo ( $A=31 \mathrm{~km}$ ca.), comp. EW.

Fig. 21a. - Somplago $(\Delta=38 \mathrm{~km}$ ca.) comp. SE-NW.
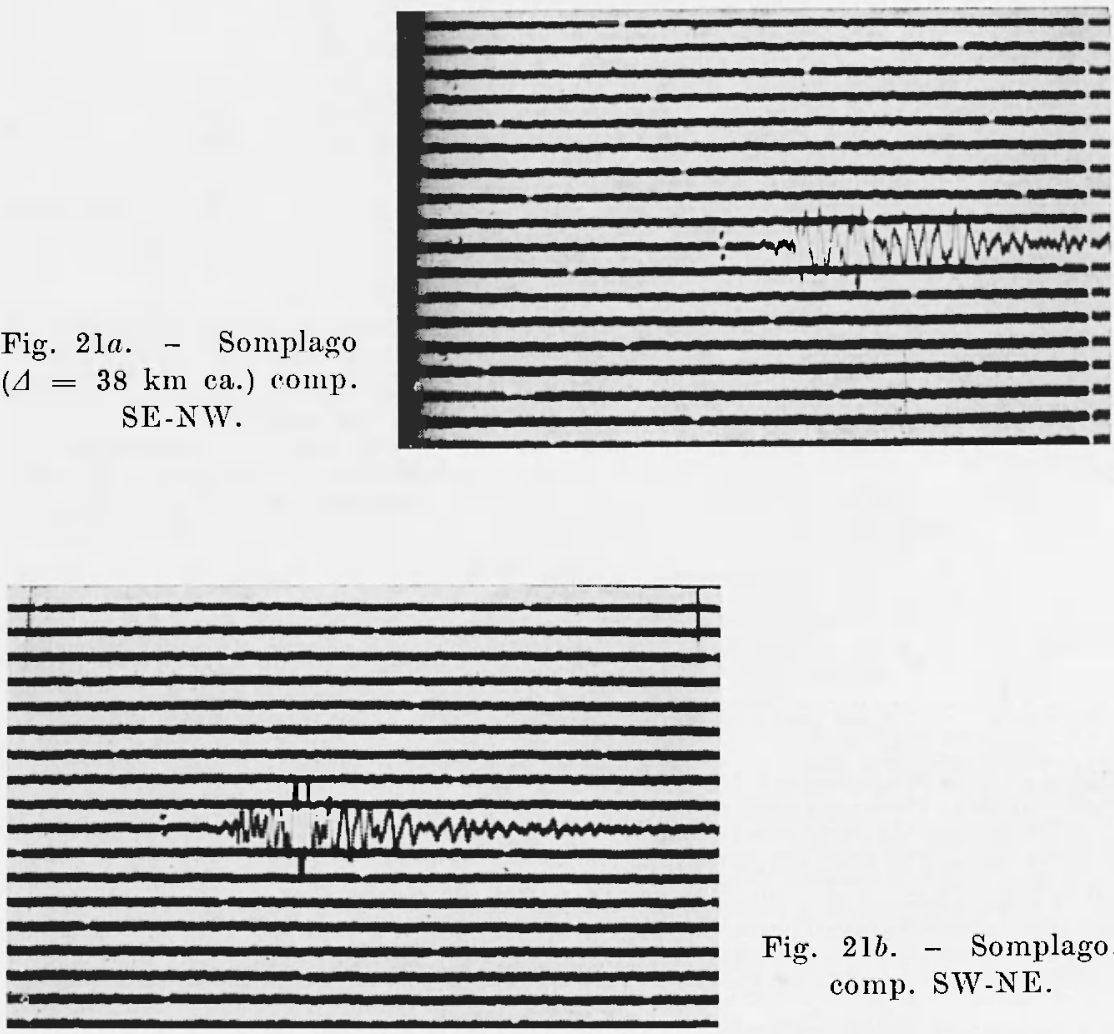

Fig. 21b. - Somplago, comp. SW-NE.

Osservazione come in Fig. 20. 
Fig. 22. - Scossa del 19.V. $1963\left(22^{\mathrm{h}} 12^{\mathrm{m}}\right.$ ca. $)$ registrata presso le stazioni sismiche di: Tolmezzo, comp. EW.
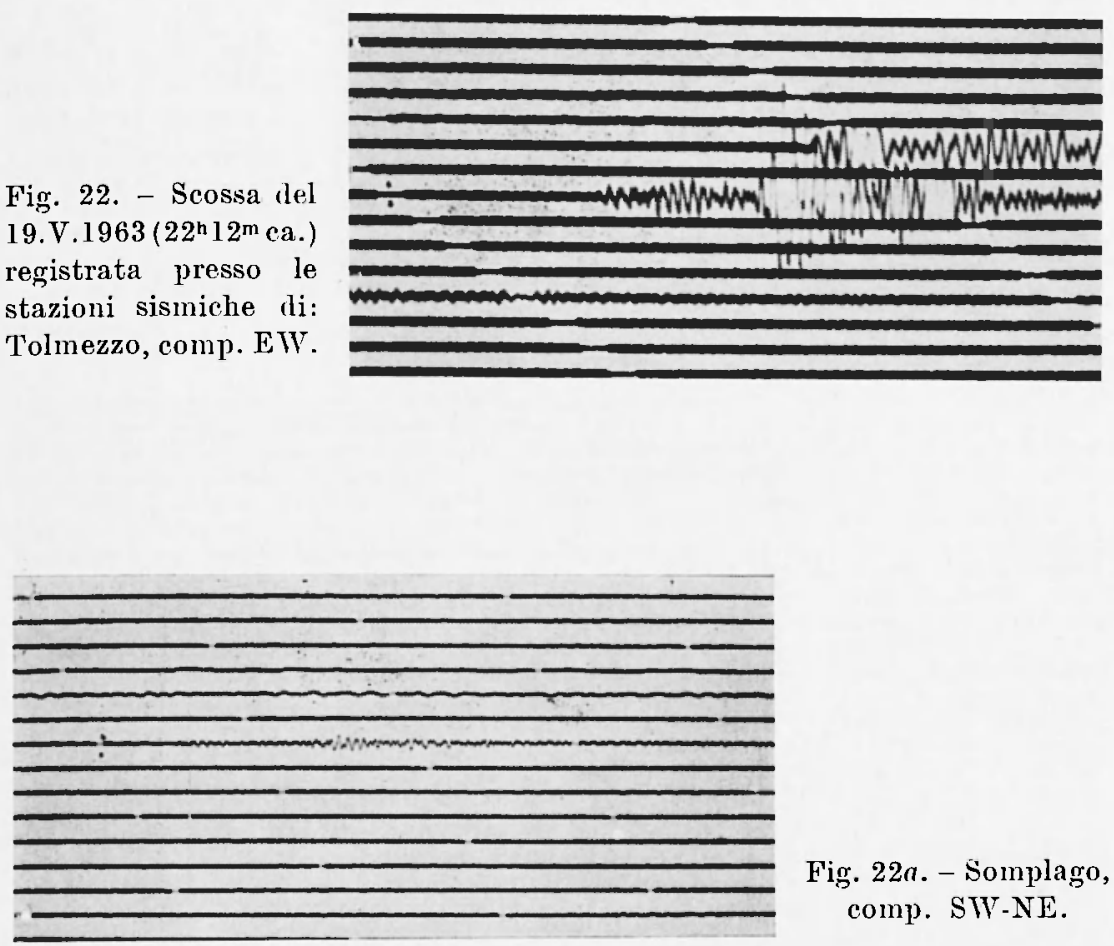

Fig. 22a. - Somplago, comp. SW-NE.

Fig. 22b. - Somplago, comp. SE-NW.

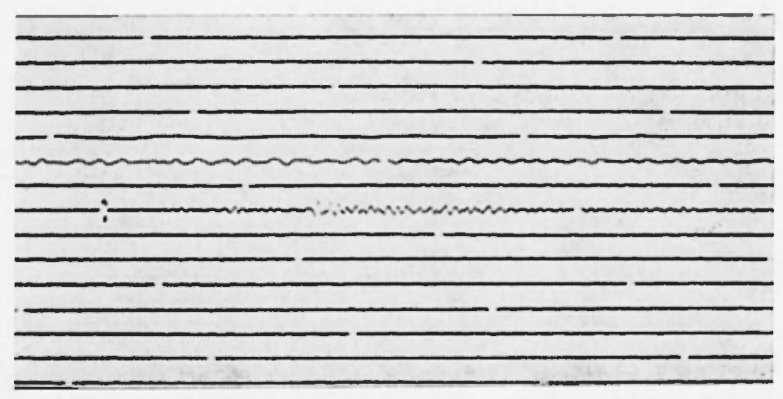

Altro esempio a testimonianza del forte "Untergrundfaktor" di Tolmezzo. 


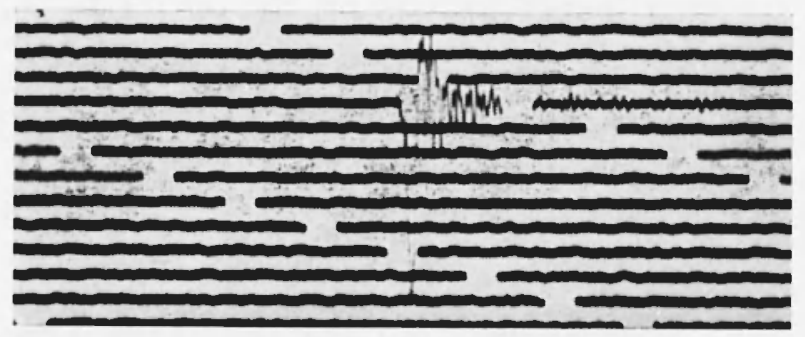

Fig. 23. - Scossa

del 7 . XII . 1963

$\left(04^{\mathrm{h}} 25^{\mathrm{m}}\right.$ ca.) registrata presso le sta.

zioni sismiche di: Tolmezzo, comp. verticale.

Fig. 23a. - Somplago, comp. verticale.
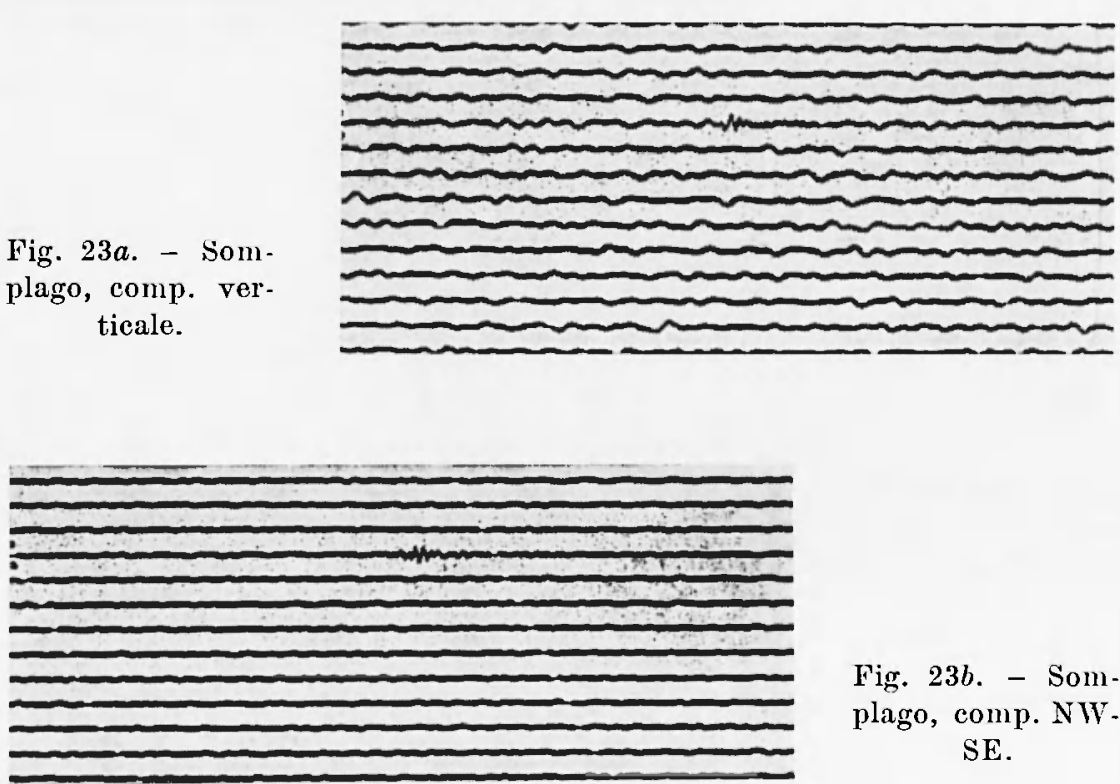

Fig. 23b. - Somplago, comp. NW. SE.

Fig. 23c. - Somplago, comp. NE. SW.

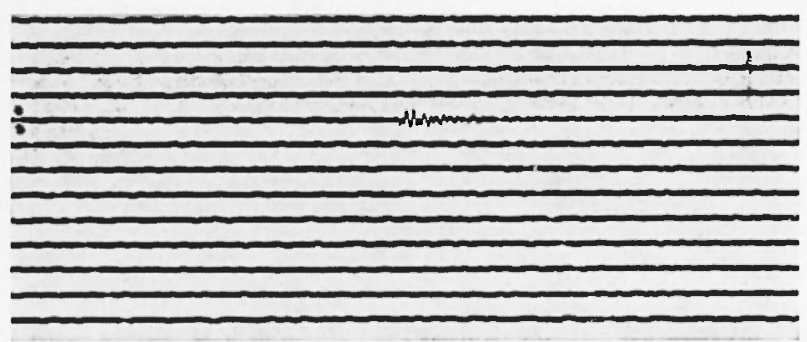

Conclusione analoga a quella della figura precedente. 
Fig. 24. - Scossa del 16. XII. $1963\left(00^{\mathrm{h}} 49^{\mathrm{m}}\right.$ ca.) registrata presso le stazioni sismiche di: Tolmezzo, comp. verticale.
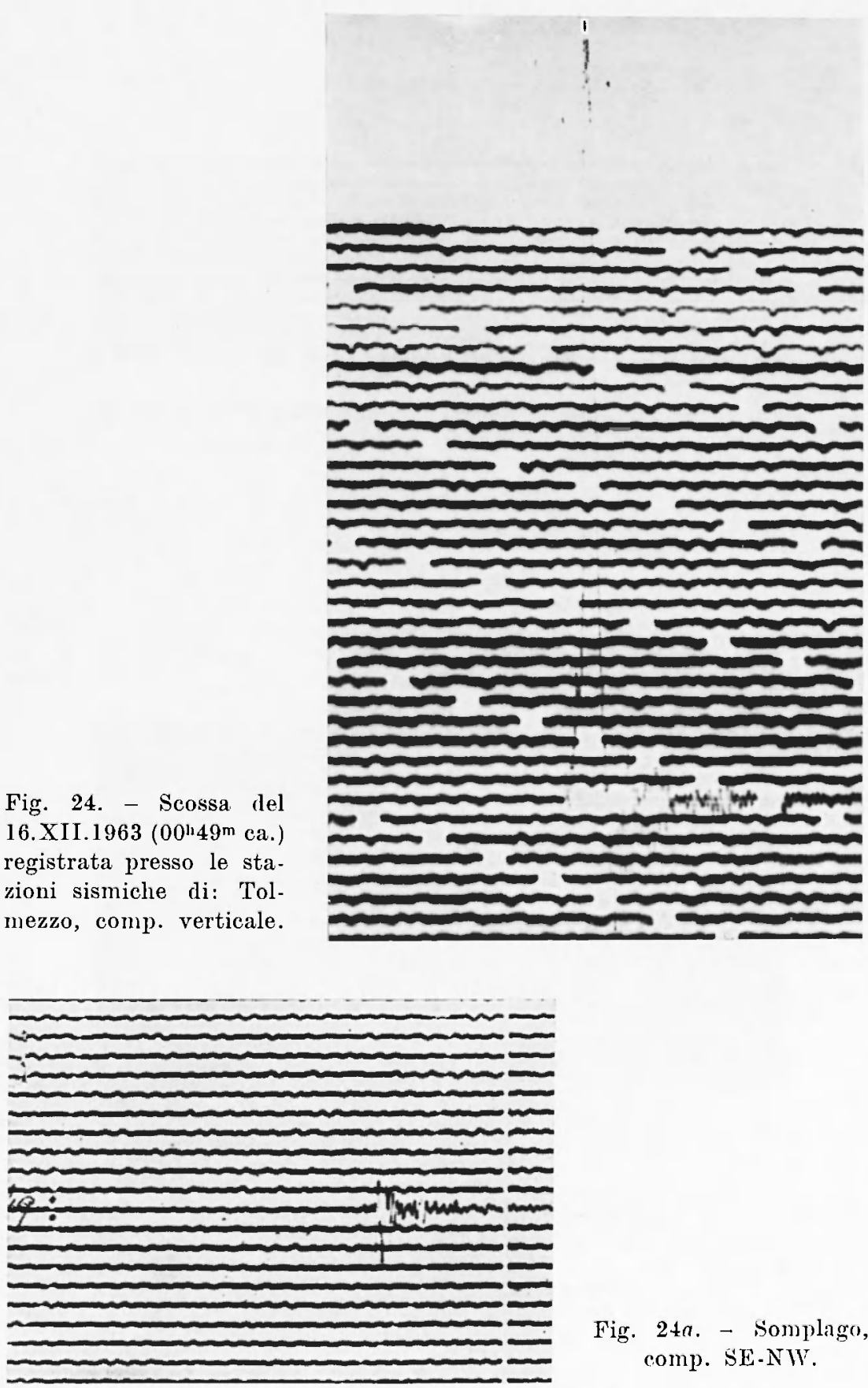

Fig. 24a. - Somplago, comp. SE-NW.

Epicentro come da [Fig. 19. 


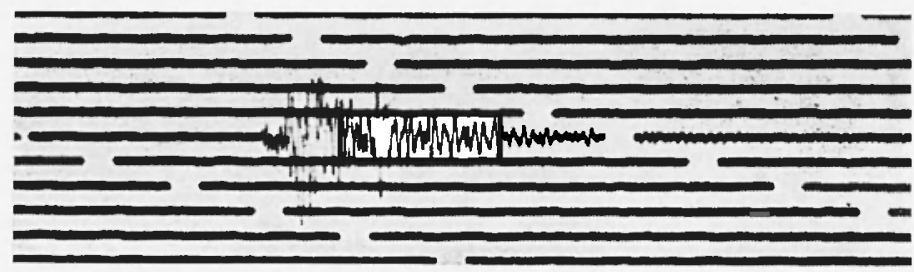

Fig. 25. - Scossa del 1.I.1964 (12 $12^{\mathrm{l}} 04^{\mathrm{m}}$ ca. $)$ registrata presso le stazioni di: Tolmezzo, comp. verticale.

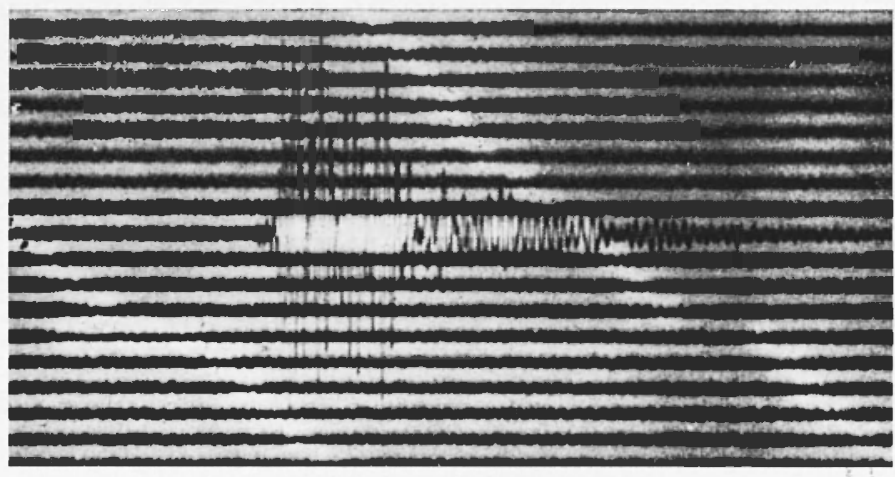

Fig. 25a. - Tolmezzo, comp. EW. 


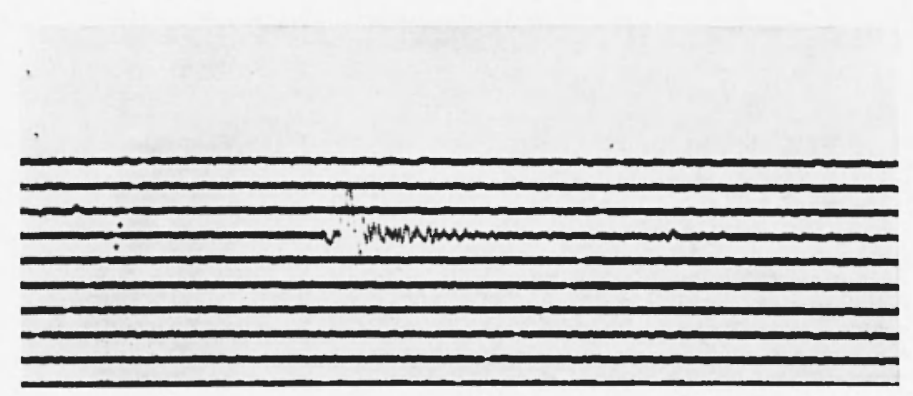

Fig. 25b. - Somplago, comp. NE-SW.

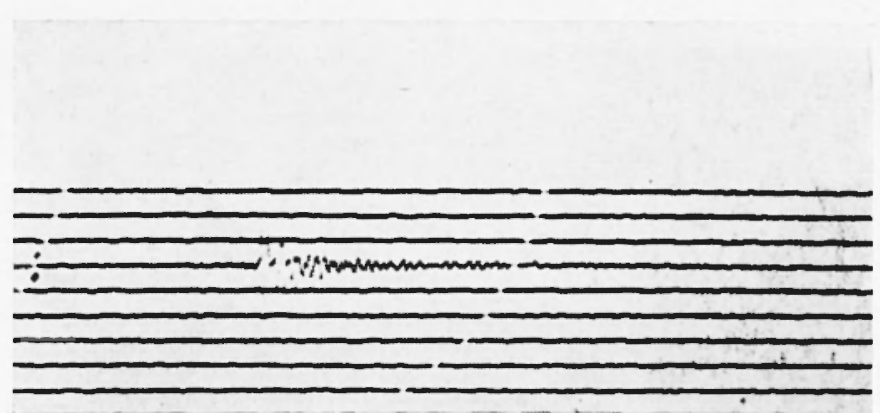

' Fig. 25c. - Somplago, comp. SE-NW.

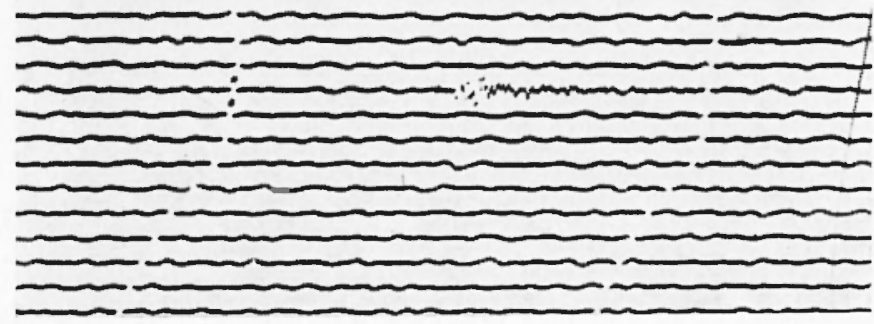

Fig. 25d. - Somplago, comp. verticale.

Epicentro come da Fig. 19. Ulteriore esempio della cospicua diversità dell'" Untergrundfaktor " nelle due vicine stazioni sismiche. 


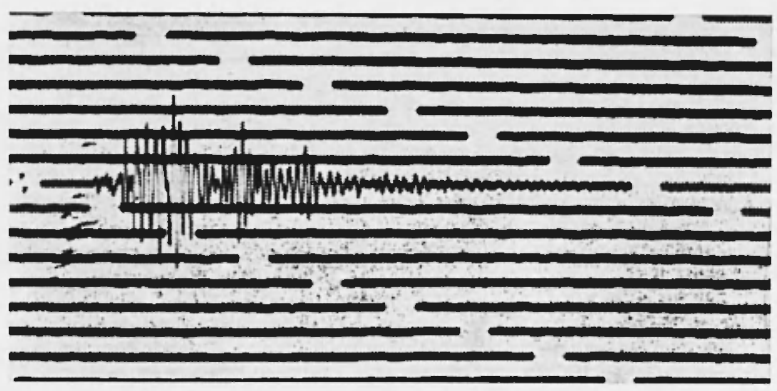

Fig. 26. - Scossa del 12.1.1964 $\left(19^{\mathrm{h}} 09^{\mathrm{m}}\right)$ registrata presso le stazioni sismiche di: Tolmezzo, comp. verticale.

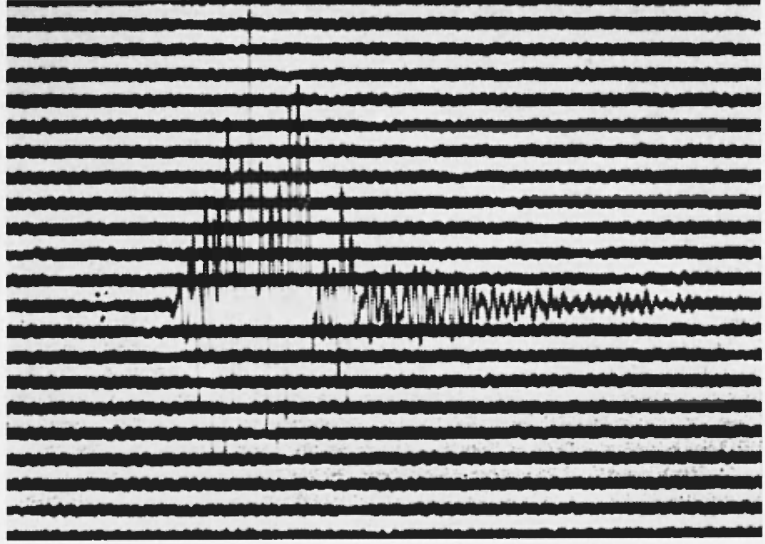

Fig. 26a. - Tolmezzo, comp. EW. 


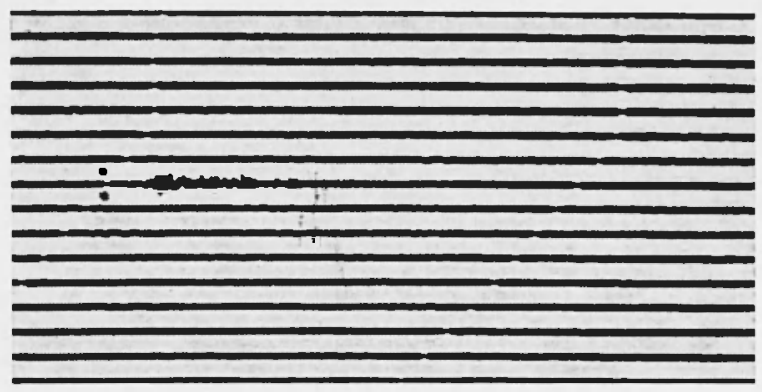

Fig. 26b. - Somplago, comp. NE-SW.

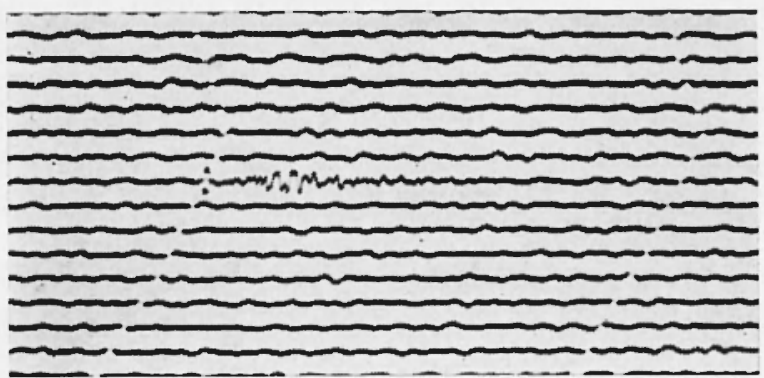

Fig. 26c. - Somplago, comp. verticale.

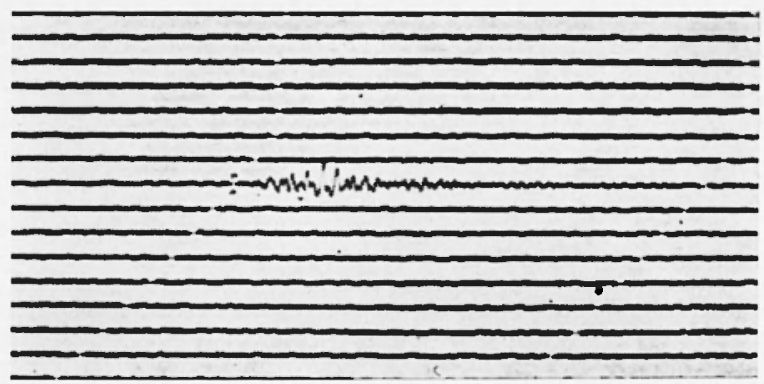

Fig. 26d. - Somplago, comp. NW-SE.

Epicentro come da Fig. 19. Conclusioni di confronto analoghe a quelle della Fig. 25. 


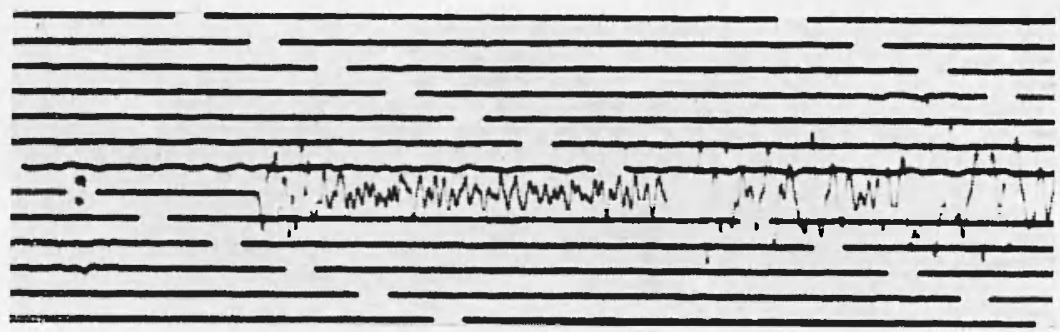

Fig. 27. - Scossa del 28, I. $1964\left(15^{\mathrm{h}} 17^{\mathrm{m}} \mathrm{ca}\right.$.) registrata nelle stazioni sismiche di: Tolmezzo, comp. verticale.

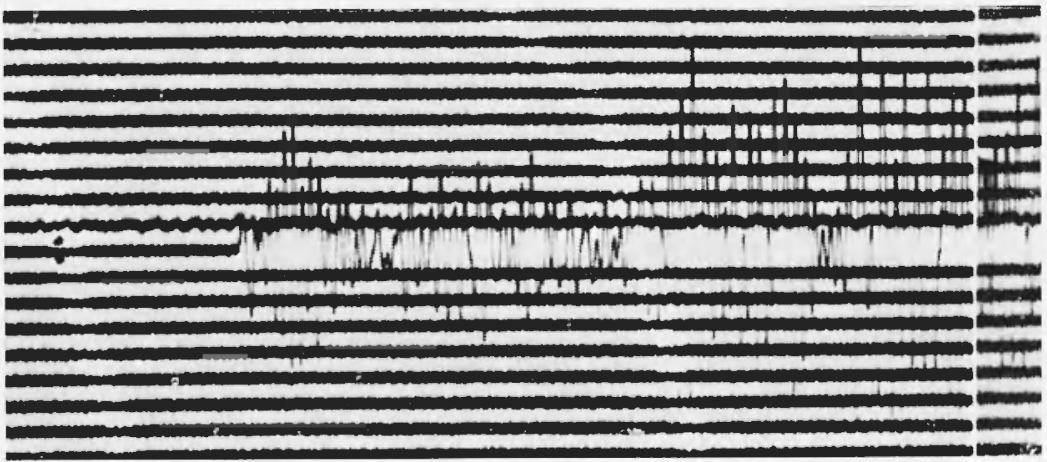

Fig, 27a. - Tolmezzo, comp. EW.

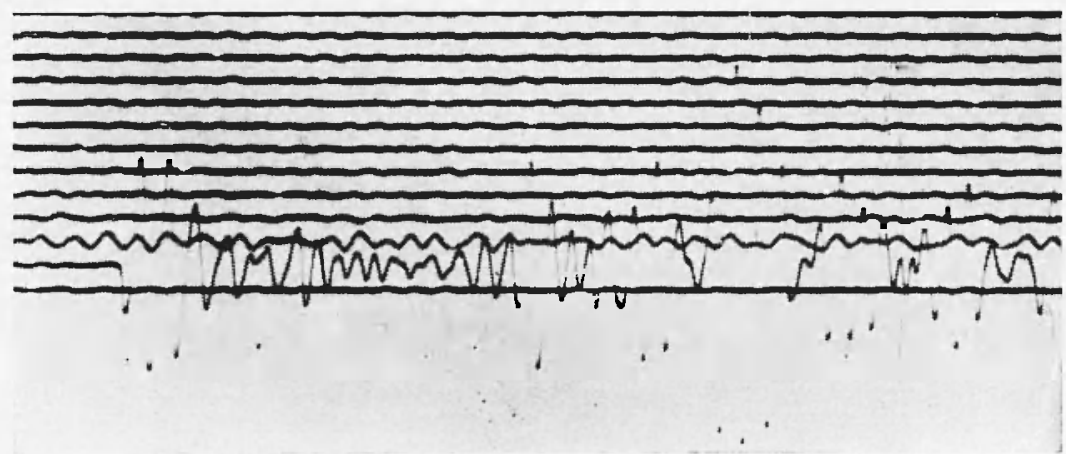

Fig. 27b. - Somplago, comp. NW-SE.

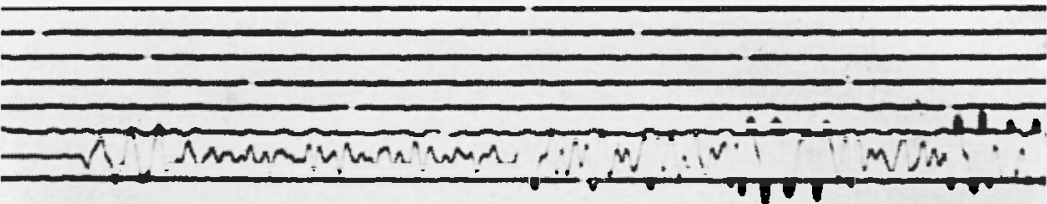

Fig. 27c. - Somplago, comp, verticale.

Epicentro nell'Indu-Kush. Anche per terremoti lontani, la stazione sismica di Tolmezzo esalta le vibrazioni a più breve periodo. 
c) Non sempre le variazioni della verticale coincidono con periodi di attività sismica nella regione. Ciò può essere spiegato tenendo conto del fatto che l'agitazione tettonica di una regione non deve necessariamente manifestarsi in attività sismica, che si ha soltanto quando il mezzo perviene al limite di rottura. Periodi caratterizzati da movimenti della verticale, non seguiti immediatamente da scosse sismiche, corrispondendo ad una concentrazione dell'energia in profondità, potrebbero anzi preludere a scosse di maggior intensità in periodi successivi. I movimenti sismici più forti sembra si possano in sostanza verificare solo se preceduti da quiete sismica corrispondente peraltro a movimenti orogenetici in atto, rivelati in superficie da variazioni della verticale.

Per quanto riguarda l'agitazione clinografica non accompagnata da attività sismica - come è avvenuto nell'inverno 62-63 —, essa può essere conseguenza di lenti movimenti, attraverso i quali viene gradualmente liberata l'energia, destinata altrimenti ad accumularsi in profondità. Tale funzione è senz'altro svolta dai bruschi fremiti degli strati rocciosi rivelati dall'inquietudine; detto fenomeno infatti coincide per lo più con un numero di scosse minore (e di minore intensità) che in altri periodi di attività sismica.

d) Sembra dunque possibile affermare che le deviazioni della verticale non presuppongono necessariamente una attività sismica contemporanea o susseguente; al contrario, i movimenti sismici sono sempre preceduti o coincidono con movimenti della verticale apparente nella "strained region".

Con tale affermazione non contrasta la constatazione che nel periodo esaminato si registrino spesso a Tolmezzo delle scosse in momenti di assoluta quiete clinografica, come è accaduto per le scosse fra il 19 e il 27 Agosto 61; quelle del Luglio-Agosto 62 e di Aprile, Maggio e Luglio 63. Va infatti notato che presso la stazione di Tolmezzo era in funzione la sola componente $\mathrm{EW}$, e pertanto nel prendere in esame le registrazioni si è tenuto conto di tutte le scosse avvenute in un certo raggio. E dunque logico supporre che alcune delle scosse considerate possano non aver alcun legame con le variazioni della verticale registrate presso l'Ambiesta, avendo avuto origine in zone indipendenti dal punto di vista geodinamico.

E stato già detto che l'origine di una delle scosse dell'Agosto 62, mentre i clinografi erano in quiete deve probabilmente essere localizzata presso Villa Santina, situata sulla sponda sinistra del Tagliamento.

Il 28 Luglio 63, si è registrata a Tolmezzo una scossa di notevole intensità, di cui non si è avuta traccia sui clinografi; dalle registrazioni di 
Somplago (Fig. 18), dove l'intensità è risultata molto minore, sembra che tale scossa abbia avuto origine in una zona situata a circa $9 \mathrm{~km}$ in direzione pressoché $\mathrm{NW}$, ovvero sulla sponda sinistra del Tagliamento. Il potente ammasso di materiale alluvionale che riempie la valle del Tagliamento, presumibilmente per notevoli profondità, probabilmente assorbe gran parte dell'energia sismica; e ciò spiega la notevole differenza di intensità con cui la scossa suddetta è stata registrata nelle stazioni di Somplago e Tolmezzo.

Per questa scossa, i clinografi dell'Ambiesta possono essersi trovati al di fuori della zona sottoposta a deformazione, da ritenersi molto piccola, data la modesta intensità delle scosse stesse.

6. - Sui limiti della "strained region " devono, ad ogni modo, influire anche le grandi dislocazioni tettoniche di una regione: una faglia puó infatti bruscamente limitare la zona in cui si risentono le deformazioni superficiali connesse con i movimenti sismici. Proprio presso l'Amhiesta si ha l'esempio di come due zone, pur situate a poca distanza l'una dall'altra, per effetto di una linea di frattura abbiano deformazioni superficiali differenti.

La zona in esame comprende parte delle Prealpi Carniche e parte delle Alpi Carniche, ergentisi rispettivamente a sud e a nord della depressione dell'alto Tagliamento; in tutta la regione prevalgono i terreni calcarei mesozoici che nelle Prealpi appaiono in una serie di pieghe con direzione est-ovest, molto compresse, rovesciate verso sud e in parte stirate in pieghe-faglie, cosicché ne risulta una struttura a scaglie embriciate $\left(^{3-4}\right)$. L'ultima scaglia è limitata a Nord da una grande flessura, che corre lungo l'alto Tagliamento e che nel tratto Villa Santina-Tolmezzo si è certamente convertita in faglia (Fig. 19). A nord della faglia-flessura del Tagliamento, le linee di dislocazione a direzione est-ovest si incrociano con un altro sistema a direzione prevalente nord-sud.

La presenza lungo il Tagliamento di una faglia-flessura, accompagnata da faglie secondarie potrebbe quindi spiegare perché presso l'Ambiesta non si verifichino deviazioni della verticale in corrispondenza di scosse con ipocentro sulla sponda sinistra del Tagliamento.

D'altro canto, la regione a sud del Tagliamento, date le sue caratteristiche tettoniche generali, deve probabilmente essere suddivisa in più zone separate fra loro da piani di scorrimento con direzione est-ovest; in tal modo, anche quelle scosse che sono state localizzate a sud dell'Ambiesta verrebbero ad avere la loro origine in zone geodinamicamente indipendenti da quella in cui sono situati i clinografi. 
7. - A conclusione di queste brevi note, che hanno sostanzialmente carattere preliminare, è da sottolineare un'altra caratteristica della sismicità nella zona intorno a Tolmezzo: il notevole contrasto della risposta elastica del mezzo, allorché si passa da Tolmezzo - nella valle del Tagliamento - alla nuova stazione sismica di Somplago, $7 \mathrm{~km}$ circa a SE, situata nella caverna della centrale idroelettrica ricavata in roccia, presso il lago di Cavazzo.

A Tolmezzo, le oscillazioni a più breve periodo vengono fortemente esaltate dal sottosuolo sabbioso, costituito dal letto del Tagliamento, cui si sono successivamente sovrapposti i detriti di erosione del Monte Amariana, alla cui base si è formata una potente conoide, dopo il crollo della primitiva cima dell'Amariana stessa. Ciò è già stato osservato in un precedente lavoro $\left(^{5}\right)$. Da quando però ha iniziato il funzionamento la stazione sismica di Somplago, le registrazioni di detta stazione hanno fornito ulteriori, chiare conferme di questo fatto. Tutti i terremoti di carattere locale, provocano a Tolmezzo registrazioni di ampiezza nettamente superiore a quella dei sismogrammi registrati a Somplago, con apparecchi aventi le stesse caratteristiche. Vedansi, al riguardo, le Figg. 20-26. Questo vale anche per i terremoti di lontana origine, almeno per quanto concerne le fasi iniziali (Fig. 27).

Ecco perché a Tolmezzo vengono normalmente registrate fasi, che sfuggono ad apparecchi - con uguali caratteristiche od anche più potenti

- di altre stazioni sismiche.

\section{BIBLIOGRAFIA}

(1) Calor P., About some Phenomena preceding and following the seismic Movements in the Zone characterized by high Seismicity. "Contributions in Geophysics: in Honor of Beno Gutenberg ". Pergamon Press-London, 1958.

(2) Calor P., Moti lenti ed improvvisi nella crosta terrestre e loro reciproche relazioni. "Scientia ", 56, 1 (1962).

${ }^{(3)}$ Gontani M., Il terremoto friulano del 27 .1Larzo 1928 nei suoi rapporti con la tettonica. "Giornale di Geologia ", III (1928).

${ }^{(4)}$ Selli R., Schema geologico delle Alpi Camiche e Giulie occidentali. "Soc. Geologica Italiana - LXII Adunanza estiva"(1963).

$\left({ }^{5}\right)$ Calor P., Seismic Waves from the Outer and the Inner Core. "Geophys. Journ. of the Roy Astr. Soc. "4, (1961). 